\title{
Genetic and metabolic factors: the perfect combination to treat metabolic associated fatty liver disease
}

\author{
Marica Meroni ${ }^{1,2 \dagger}$, Miriam Longo ${ }^{1,3 \dagger}$, Paola Dongiovanni ${ }^{1^{*}}$ \\ ${ }^{1}$ General Medicine and Metabolic Diseases, Fondazione IRCCS Ca' Granda Ospedale Maggiore Policlinico, 20122 Milano, Italy \\ ${ }^{2}$ Department of Pathophysiology and Transplantation, Università degli Studi di Milano, 20122 Milano, Italy \\ ${ }^{3}$ Department of Clinical Sciences and Community Health, Università degli Studi di Milano, 20122 Milano, Italy
}

${ }^{\dagger}$ These authors contributed equally to the work.

*Correspondence: Paola Dongiovanni, General Medicine and Metabolic Diseases, Fondazione IRCCS Cà Granda Ospedale Maggiore Policlinico, Via Francesco Sforza 35, 20122 Milano, Italy. paola.dongiovanni@policlinico.mi.it

Academic Editor: Lindsay A. Farrer, Boston University School of Medicine, USA

Received: May 28, 2020 Accepted: June 20, 2020 Published: August 31, 2020

Cite this article: Meroni M, Longo M, Dongiovanni P. Genetic and metabolic factors: the perfect combination to treat metabolic associated fatty liver disease. Explor Med. 2020;1:218-43. https://doi.org/10.37349/emed.2020.00015

\begin{abstract}
The prevalence of nonalcoholic or more recently re-defined metabolic associated fatty liver disease (MAFLD) is rapidly growing worldwide. It is characterized by hepatic fat accumulation exceeding $5 \%$ of liver weight not attributable to alcohol consumption. MAFLD refers to an umbrella of conditions ranging from simple steatosis to nonalcoholic steatohepatitis which may finally progress to cirrhosis and hepatocellular carcinoma. MAFLD is closely related to components of the metabolic syndrome and to environmental factors. In addition to the latter, genetic predisposition plays a key role in MAFLD pathogenesis and strictly contributes to its progressive forms. The candidate genes which have been related to MAFLD hereditability are mainly involved in lipids remodeling, lipid droplets assembly, lipoprotein packaging and secretion, de novo lipogenesis, and mitochondrial redox status. In the recent years, it has emerged the opportunity to translate the genetics into clinics by aggregating the genetic variants mostly associated with MAFLD in polygenic risk scores. These scores might be used in combination with metabolic factors to identify those patients at higher risk to develop more severe liver disease and to schedule an individual therapeutic approach.
\end{abstract}

\section{Keywords}

MAFLD, genetics, personalized medicine, polygenic risk scores

\section{Introduction}

Nonalcoholic or more recently re-defined metabolic associated fatty liver disease (MAFLD) is regarded as the most relevant liver disease of the twenty-first century, affecting at least one third of the general population [1-3] and it is predicted to become the leading cause of liver transplantation by 2030 [4]. Thus, given its pandemic proportion, MAFLD constitutes a tremendous socio-economic and health burden [5]. MAFLD is characterized by excessive hepatic fat deposition that exceeds $5 \%$ of liver weight, in the absence of alcohol intake. It embraces a variable phenotypic spectrum of hepatic disorders, spanning from isolated (C) The Author(s) 2020. This is an Open Access article licensed under a Creative Commons Attribution 4.0 International License (https://creativecommons.org/licenses/by/4.0/), which permits unrestricted use, sharing, adaptation, distribution and reproduction in any medium or format, for any purpose, even commercially, as long as you give appropriate credit to the original author(s) and the source, provide a link to the Creative Commons license, and indicate if changes were made. 
steatosis to its inflammatory form, nonalcoholic steatohepatitis (NASH), defined by lobular inflammation, hepatocyte ballooning degeneration and fibrotic processes activation. NASH may then progress towards endstage liver injuries, such as cirrhosis and hepatocellular carcinoma (HCC) [6, 7].

At epidemiological level, MAFLD is tightly correlated with excessive adiposity, type 2 diabetes (T2D) and metabolic syndrome (MetS) hallmarks [8] and its pathophysiology is closely intertwined with obesity, insulin resistance (IR) and atherogenic dyslipidemia [9]. Hence, it is referred to as the hepatic manifestation of MetS and unhealthy dietary habits, fructose over-consumption and sedentary lifestyle may represent pivotal environmental risk factors for its pathogenesis $[10,11]$.

However, as a complex disease, MAFLD has a multi-factorial pathogenesis and the individual susceptibility to develop it may be partly attributable to inherited risk factors [12]. Hitherto, the best-known MAFLD inherited components are represented by single nucleotide polymorphisms in genes regulating hepatic lipid remodeling, turn-over and dismissal, among which patatin-like phospholipase domain-containing 3 (PNPLA3), transmembrane 6 superfamily member 2 (TM6SF2), membrane bound o-acyltransferase domaincontaining 7 (MBOAT7), and Glucokinase regulator (GCKR) [12]. Nonetheless, only a minor percentage of interindividual variability is explained by these common mutations and the majority of the phenotypic differences in hepatic manifestations may also derive from gene-environment interaction, due to hereditable epigenetic modifications $[13,14]$. Furthermore, among the plethora of multiple parallel hits, a new perspective in the pathophysiology of fatty liver is represented by the crucial role of intestinal dysbiosis, microbial metabolites and enhanced intestinal permeability $[15,16]$.

Nowadays, liver biopsy remains the gold standard procedure for diagnosis of MAFLD and no therapeutic consensus exists for the treatment of MAFLD patients $[10,11]$. However, the deep-knowledge of the genetic landscape responsible for the individual susceptibility towards MAFLD and its progressive forms may open the possibility to identify score-based strategies of combined genetic risk factors for liver damage prediction and pave the way to personalize therapeutic approaches. In addition, the study of the interaction between environment and inherited factors may be also useful to address specific clinical tailored advices, health practices for disease prevention and genome-based dietary guidelines [10,11].

For this reason, this review aimed to focus on the relevance of the assessment of the individual genetic make-up in order to ameliorate disease diagnosis, by using non-invasive strategies and to individualize the clinical management of MAFLD patients, avoiding its progression towards end-stage conditions.

\section{MAFLD is an inheritable disease}

In the past years, it has been widely demonstrated that IR and obesity are the most prevalent risk factors of MAFLD pathogenesis. However, among subjects with the same grade of adiposity, there is a substantial variability in hepatic lipid deposition, raising the possibility that several other risk factors may participate to steatosis development. Familial, twin and epidemiological studies indicate that hepatic fat accumulation has a strong inherited component $[17,18]$. In particular, Schwimmer et al. [19], revealed that parents and siblings of overweight children with MAFLD display an enhanced susceptibility to develop fatty liver compared to obese children without MAFLD. Even more, a familial NASH clustering is common, reaching $18 \%$ of patients having a similarly affected first degree relative [20].

Twin studies demonstrated that approximately $60 \%$ of the variation in serum alanine aminotransferase (ALT) as well as in circulating insulin concentrations, are genetically determined in absence of alcohol abuse or viral hepatitis and that are both significantly correlated with liver fat content [21]. Moreover, Loomba et al. [19], demonstrated that hepatic steatosis, assessed by using magnetic resonance imaging proton-density fat fraction (MRI-PDFF) and hepatic fibrosis, based on stiffness determined by MR elastography, are both strictly correlated in monozygotic twins compared to dizygotic twins. In a multivariable model, adjusting for age, gender and ethnicity, the percentages of hereditability of hepatic steatosis and fibrosis were attested at $52 \%$ and 50\%, respectively [22]. In addition, in the same cohort of twins, Cui et al. [23], revealed a high level of shared genetic components between hepatic steatosis and fibrosis. 
Even the ethnicity can influence the predisposition towards MAFLD $[17,18]$. In two large multi-ethnic population studies conducted in the United States, it has been demonstrated that Hispanic individuals have an higher risk to develop MAFLD than those of European descent [24, 25]. More specifically, intra-ethnic differences exist and amongst Hispanics, Mexicans have much higher prevalence of MAFLD compared to Dominican or those from Puerto Rico [26]. On the contrary, African-Americans are protected irrespectively of diabetes, excess in body weight and socioeconomic factors, supporting the notion that heritability may exert a crucial role in MAFLD pathophysiology [12]. In particular, African-Americans differed in the metabolic response to obesity and IR when compared to either Hispanics or Caucasians, appearing more resistant to triglyceride (TG) accumulation both in the adipose tissue and in the liver [27].

Several inherited risk factors have been identified as modifier of genetic predisposition to develop MAFLD and its progressive forms [12]. In particular, Dongiovanni et al. [28], demonstrated that hepatic fat accumulation represents the main driver of the progression to end-stage liver damages in genetically predisposed individuals. Therefore, the effect of each genetic variation on the spectrum of MAFLD is closely intertwined with their ability to induce fat accumulation [28]. Nowadays, the major common predictors of the inherited predisposition to severe MAFLD are the variants in PNPLA3, TM6SF2, MBOAT7 and GCKR genes. However, given the complex genetic architecture of MAFLD, a huge number of other genetic risk factors has been identified, and score-based strategies which evaluate polygenic determinants of MAFLD seems to be the most highly predictive and they can be exploited to improve diagnostic accuracy and to guide treatment options $[29,30]$.

\section{PNPLA3: the best predictor of inheritable MAFLD}

To date, the major genetic predictor of the inter-individual and ethnicity-related differences in hepatic fat content and the main risk factor of increased susceptibility to develop progressive MAFLD is the rs738409 C $>\mathrm{G}$ variant in PNPLA3 gene (also referred to as adiponutrin), encoding the amino acid substitution isoleucine to methionine at the position 148 (p.I148M) [31]. It was firstly identified by a genome-wide association study (GWAS) in 2008 which evaluated North American population of diverse ethnicity and revealed that the prevalence of the G at-risk allele is higher in Hispanics (49\%) than in Europeans (23\%) and less frequent in African Americans (17\%), thereby explaining the inter-ethnic susceptibility to MAFLD [31]. PNPLA3 encodes a 481-amino acid membrane lipase, mainly localized in the endothelial reticulum (ER) and at the lipid droplet surface in hepatocytes, adipocytes and in hepatic stellate cells (HSCs) [32, 33]. At transcriptional level, PNPLA3 expression is controlled by the activation of the sterol regulatory element-binding protein 1 (SREBP1c)/liver $\mathrm{X}$ receptor and by Carbohydrate response element binding protein pathways, induced by post-prandial or pathological hyperinsulinemia. Moreover, PNPLA3 protein is post-transcriptionally modified by the presence of fatty acids, hindering its degradation [34, 35]. Thus, an interplay between PNPLA3 and environmental factors exists. In particular, high-sucrose and high-fructose consumption, sedentary lifestyle habits and excessive adiposity trigger the detrimental effect of p.I148M both in adults and children [36-38]. Indeed, the associations between the rs738409 variant and MAFLD may be unmasked by the increased adiposity, enhancing the genetic risk [36]. Nonetheless, it has been demonstrated that this variation constitutes a risk factor for MAFLD development and progression towards NASH and fibrosis, even in lean subjects (BMI $<25$ $\mathrm{kg} / \mathrm{m}^{3}$ ) [39].

In vitro studies demonstrated that PNPLA3 hydrolyzes lysophosphatidic acid to phosphatidic acid, and the p.I148M inherited variation strongly affects its function [40, 41]. As a consequence of the loss of PNPLA3 enzymatic activity, patients carrying $\mathrm{G}$ alleles have elevated plasma liver enzymes and a strong predisposition towards the entire spectrum of liver damage related to fatty liver, encompassing $\mathrm{NASH}$, severe fibrosis and HCC, even influencing the response to therapeutic approaches [42].

However, the physiological implication of PNPLA3 in lipid metabolism remains to be fully elucidated. In particular, neither Pnpla3 genetic deficiency nor Pnpla3 wild-type over-expression in mice induces hepatic steatosis development [41, 43, 44], whereas Pnpla3 I148M knock-in mice are characterized by fatty-laden hepatocytes upon a steatogenic high-sucrose diet challenge [45]. In these mice, Pnpla3 silencing through 
antisense oligonucleotides ameliorated all histological features of MAFLD, including liver fibrosis [46]. More specifically, it has been recently proposed that the pathogenic effect of PNPLA3 variant is mediated by its interfering with the activity of the adipose triglyceride lipase (ATGL)/patatin-like phospholipase domaincontaining 2 (PNPLA2), which is the main TG lipase in liver and adipose tissue, directly by interacting with its cofactor, namely comparative gene identification-58 (CGI-58) [47]. Indeed, overexpression of Pnpla3 I148M increased hepatic TG levels in wild-type, but not in Cgi-58 knock-out (KO) mice, suggesting that the PNPLA3 mutation may promote steatosis through a CGI-58-dependent inhibition of ATGL/PNPLA2 on lipid droplets. Superimposable results have been obtained in brown adipocytes [48]. In addition, it has been demonstrated that the p.I148M variant is able to abolish PNPLA3 ubiquitylation and proteasomal degradation resulting in the accumulation of PNPLA3 I148M, without altering lipid synthesis [49]. Therefore, the likely mechanisms through which the p.I148M variant induces intracellular fat deposition seems to be due to the accumulation of the PNPLA3 mutated protein on the lipid droplet surface due its reduced ubiquitination, thus impairing TG mobilization even by other lipases (i.e. ATGL/PNPLA2) and hampering TG turnover and dismissal $[49,50]$. The proof of this concept is widely explained by BasuRay et al. [51], which engineered a synthetic isoform of PNPLA3 that uncouples protein accumulation from loss of enzymatic activity. These authors demonstrated that the expression of an ubiquitylation-resistant form of Pnpla3 in mice is responsible for the accumulation of Pnpla3 mutated protein on hepatic lipid droplets. Furthermore, the reduction of Pnpla3 expression by shRNA or by or proteolysis-targeting chimera (PROTAC)-mediated degradation dampened TG storage formation in mice overexpressing Pnpla3 I148M [51]. In keeping with these findings, PNPLA3 E434K variant ameliorates the impact of the p.I148M on steatosis development in MAFLD patients, whereby down-modulating PNPLA3 expression on the lipid droplets [50]. Another potential mechanism that explain TG accumulation in presence of the p.I148M variant is related to the impairment of lipophagy in hepatocytes, thus hampering autophagic fluxes and lipid droplet degradation [52].

A lipidomic analysis, provided by Luukkonen et al. [53], demonstrated that TG in very-low density lipoproteins (VLDL) are depleted of polyunsaturated fatty acids (PUFAs) in I148M homozygous subjects both under fasting and postprandial conditions. As a consequence, in I148M hepatic cells, PUFA incorporation into TG is increased and PUFA-containing diacylglycerols (DAGs) are accumulated, at the expense of phosphatidylcholines (PCs) [53]. The hepatic lipid composition of DAG species may, in turn, strongly affect insulin sensitivity. However, the alteration in hepatic DAG levels has not confirmed by Franko et al. [54], which supported the idea that PNPLA3 variation is tightly associated with fatty liver, but not with IR, whereby dissociating these two hallmarks of MAFLD. Indeed, IR-related MAFLD is characterized by high levels of metabolically harmful saturated and mono-unsaturated TG, ceramides and free fatty acids (FFAs), whereas PNPLA3-related MAFLD by hepatic PUFA-containing TG. These observations may possibly explain why metabolic MAFLD and not PNPLA3-related MAFLD, is tightly correlated with increased risk of T2D and cardiovascular events [55]. Likewise, the excess of PUFA-containing lipids has been observed even in the adipose tissue of PNPLA3 I148M carriers, where this variant did not alter the rate of lipolysis or the composition of fasting serum FFAs [56].

Intriguingly, PNPLA3 mutated protein in HSCs exerts a detrimental effect on retinol secretion [57], directly participating to fibrogenesis and carcinogenesis, therefore, enhancing cirrhosis and HCC risks, irrespective of the predisposition to steatosis [58-61]. Indeed, MAFLD patients carrying the G allele display a distinctive histological pattern, characterized by enhanced steatosis and portal inflammation accompanied by a prominent proliferation of hepatic progenitor cell, extensive ductular reaction, HSCs and myofibroblasts activation, inducing even portal fibrosis generation and severe systemic oxidative stress [62]. Therefore, the size effect of the I148M variant on the risk of MAFLD is the strongest ever reported for a common variant [10].

Notably, this variation has been also associated with hepatic decompensation and liver-related death in a recent Italian prospective study [63], with increased risk of fibrosis progression and HCC even in patients affected by viral hepatitis or alcoholic liver disease (ALD) and with poor prognosis in patients affected by autoimmune hepatitis, independently of steatosis [64-66]. 


\section{TM6SF2 loss-of-function in MAFLD: a riddle still to be unraveled}

In 2014, an exome-wide association study conducted by Kozlitina et al. [67], in more than 80, 000 participants of three independent cohorts identified the missense rs58542926 C > T variant in the TM6SF2 gene encoding the lysine to glutamate substitution at residue 167 (E167K). Ever-increasing evidence has strengthened the role of TM6SF2 as a key regulator of cholesterol biosynthesis and lipid composition in the liver of both humans and experimental models [68-75]. TM6SF2 localizes in the ER and ER-Golgi compartments [76] and it participates to hepatic VLDL lipidation and assembly in the ER cisternae [67, 69]. In Tm6sf2 $\%$ mice, Smagris et al. [69], firstly reported that TM6SF2 is required to mobilize neutral lipids for VLDL assembly but it did not affect lipoproteins' trafficking. A lipidomic analysis in both human livers and in HuH7 human hepatoma cell line revealed that TM6SF2 silencing impairs hepatic synthesis of PC-containing PUFAs. The reduction of the unsaturated PCs pool causes an excess of free PUFAs which, in turn, inhibits VLDL assembly and favors the formation of TGs and cholesterol-esters clusters thus disturbing membrane composition and dynamics $[71,73]$. The impact of TM6SF2 deficiency has been also investigated in a fine-tuned research by 0 'Hare and colleagues who explored the role of TM6SF2 in small intestine of zebrafish and in Caco-2 enterocytes. They demonstrated that intestinal TM6SF2-deficiency induces lipid droplets accumulation, reduces lipid clearance, and promotes ER stress [77]. Interestingly, Fan and coworkers hypothesized that TM6SF2 might also exert an enzymatic activity by converting zymosterol into 5 - $\alpha$-cholesta-7,24-dien-3 $\beta$-ol, a critical step in cholesterol biosynthesis, although further studies will be crucial to corroborate this finding [70].

In presence of the 167Lys substitution, TM6SF2 expression is 50\% reduced in the HuH7 hepatocytes and generated a TM6SF2 misfolded protein which may run into rapid intracellular turnover and degradation further causing its hepatic down-regulation [67]. In addition, Tm6sf2 knock-down in the livers of mice resulted in 3-fold enrichment of intrahepatic TGs, probably due to VLDL retention, paralleled with decreased plasma cholesterol [67]. The E167K genetic variant was independently associated with higher circulating levels of ALT, a marker of liver damage, hepatic TG content, and MAFLD stages in both children and adults $[67,77-82]$. The minor $\mathrm{T}$ allele was also correlated with lower serum cholesterol and TGs levels in several cohorts of MAFLD patients [77,81] and in large population studies including the Dallas Heart Study, the Dallas Biobank and the Copenhagen Study [67]. In another exome-wide association study encompassing 300, 000 participants, the authors identified 444 coding and noncoding genetic variants associated with plasma lipids and, among them, the E167K TM6SF2 variant resulted as one of the causal gene that strongly influenced metabolic traits as it mitigated circulating TG levels and increased risk of fatty liver and T2D [83]. Intriguingly, in a large cohort of 1, 201 biopsy-proven MAFLD individuals who underwent liver biopsy, carriers of the rs58542926 variant showed a higher degree of steatosis, necroinflammation, ballooning and fibrosis but were protected against cardiovascular events [81]. In keeping with these results, numerous studies revealed that the non-synonymous rs58542926 polymorphism reduces serum concentration of "bad" cholesterol enriched in low density lipoproteins (LDL) [67, 77, 79-81, 84-86]. 0'Hare et al. [77], evaluated the effects of the rs58542926 single nucleotide polymorphism (SNP) in two independent cohorts entailing 983 patients who underwent bariatric surgery and 3, 556 participants who were enrolled in the Amish Complex Disease Research Program (ACDRP). Despite the metabolic biochemical differences, carriers of E167K variant showed an improved fasting lipid profile in both case studies and they exhibited lower postprandial serum TGs after high fat challenge [77]. Therefore, it has been suggested that the T risk allele may disentangle MAFLD from cardiovascular disorders but it increases liver disease severity [81, 87]. Even Sookoian et al. [88], found a significant correlation between the $\mathrm{E} 167 \mathrm{~K}$ variant and histological steatosis in 361 subjects and such findings were consistent with those reported by Mancina et al. [78], and Dongiovanni et al. [81]. In a multiethnic pediatric cohort consisting of 957 obese individuals, of whom 459 underwent MRI for MAFLD diagnosis, the minor T allele positively associated with serum ALT levels, fatty liver and with improved lipid profile only in Caucasian and Afro-American children, thus highlighting how the solely ethnical branch may confer a different genetic predisposition to MAFLD [79]. In addition, in 60 non-obese normo-lipemic MAFLD patients, whose liver biopsies were available, the TM6SF2 at-risk genotype increased the susceptibility to both hepatic and adipose tissues IR as well as it impaired the post-prandial incretin secretion and, consequently, its effects on pancreatic $\beta$-cells [89]. Furthermore, at multivariable models, the TM6SF2 rs58542926 variant is associated 
with MAFLD even in lean individuals, who showed a higher prevalence of carriage of the T allele compared to obese patients [90].

Overwhelming data supported that TM6SF2 loss-of-function affects lipid metabolism and predisposed to hepatic steatosis development and progressive liver injury [79, 81, 82, 91, 92]. However, the association between the minor $\mathrm{T}$ allele with both fibrosis and HCC is still controversial, probably due different diagnostic tools exploited for MAFLD diagnosis (MRI, ultrasound, and liver biopsy) [74, 88, 92]. For instance, Sookoian et al. [88], did not find any associations between the TM6SF2 mutation and circulating transaminases, necroinflammation and fibrosis possibly due to the low frequency of the rs58542926 polymorphism and lack of statistical power. Conversely, a meta-analysis of 24, 147 individuals affected by heterogeneous chronic liver disorders associated the TM6SF2 rs58542926 genotype with higher susceptibility to develop MAFLD, ALD, cirrhosis, and HCC than viral hepatitis [93]. In alcohol abusers affected by alcohol-related cirrhosis, the presence of the rs58542926 C > T variant represented an additional risk factor to develop HCC [94]. According to these results, the E167K TM6SF2 did not influence fibrosis severity in 694 consecutively biopsied Caucasians with chronic hepatitis C [95] whereas it correlated with alcohol-related HCC in 511 cirrhotic patients [96] and in a prospective cohort of 249 ALD patients [97]. Liu et al. [91], reported that carriers of the T risk allele conferred an increased predisposition to develop MAFLD-related advanced fibrosis in two independent cohort regardless of other confounders as gender, sex, BMI, T2D and PNPLA3 rs738409 genotype. In 11 biopsy-proven MAFLD pediatric subjects, carriers of the TM6SF2 C > T variant displayed a higher grade of fibrosis compared to CC genotype [79]. Finally, the association among the T risk allele with hepatic fibrosis [92] and HCC risk [98] was further observed in a cross-sectional and in a small cohort study including 502 and 129 MAFLD patients, respectively.

\section{MBOAT7: a novel modifier of progressive liver disease}

In 2015, a GWAS revealed that the common rs641738 C > T variant in the MBOAT7-transmembrane channel like 4 (TMC4) locus on chromosome 19, increased the susceptibility to cirrhosis in alcoholics $[99,100]$. Then, Mancina and Dongiovanni et al. [101], demonstrated that the rs641738 variation associates with a strong predisposition towards hepatic fat accumulation and to the entire phenotypic spectrum of liver injuries related to MAFLD, including HCC [101-103]. This evidence has been further corroborated in pediatric individuals in which MBOAT7 variation is strongly correlated with enhanced ALT levels, C-reactive protein concentrations, and with increased total body fat percentage [104]. Moreover, it has been demonstrated that in pediatric MAFLD patients, T risk allele carriers were characterized by increased ALT, and more advanced steatosis and fibrosis, revealing a combined effect of the MBOAT7 rs641738, PNPLA3 I148M, and TM6SF2 E167K variants on pediatric MAFLD risk [105], even confirmed in adult patients [29, 30, 106]. Notably, the rs641738 has been also identified as a risk factor for fibrosis development even in viral hepatitis B and C, possibly representing a common modifier of liver damage $[107,108]$ and it has been shown to be involved in primary biliary cholangitis, exerting a positive effect on transplant free survival $[109,110]$. However, the association between the rs641738 variant and liver injuries remains still controversial and not fully replicated, mainly due to the different sample size and ethnicity of the cohorts enrolled in the studies or to the diverse assessment of hepatic steatosis [111-115]. Notwithstanding, a very recent meta-analysis across 42 studies, confirmed the associations between the rs641738 variant and liver fat, ALT, histological severity of MAFLD, fibrosis and HCC in individuals of European descent [116].

MBOAT7, also known as lyso-phosphatidylinositol (lyso-PI) acyl-transferase1 (LPIAT1), encodes for an enzyme member of the "Lands' Cycle" of phospholipid acyl-chain remodeling of the membranes. It is mainly localized in the membrane bridging ER and mitochondria in which the fat biosynthesis and lipid droplets formation occurs. It conjugates an acyl-CoA to the second acyl-chain of lyso-phospholipids, using arachidonoylCoA. Thus, it modulates the desaturation of phospholipids and the amount of free arachidonic acid, precursor of proinflammatory eicosanoids [117]. Mancina and Dongiovanni et al. [101], have deeply demonstrated that the mechanisms underlying the association between the rs641738 variant and liver damage is related to the hampered hepatic gene and protein expression of MBOAT7, altering in turn phosphatidylinositol 
species [118], as confirmed by Luukkonen et al. [102]. This evidence is supported by an our very recent paper, that pointed out that hepatic MBOAT7 down-regulation is a dysfunctional response to hyperinsulinemia and that its reduced expression induces intracellular fat accumulation in clinical samples, in in vivo models of MAFLD and in genetically edited HepG2 cells (MBOAT7 $\%$ ) [118]. In particular, MBOAT7 is hampered in presence of hyperinsulinemia and severe liver damage in obese patients, irrespectively of the individual genetic make-up, as well as in experimental models of obesity-related or IR-related MAFLD. An impairment in MBOAT7 function contributes to accumulate saturated phospholipids, mainly, phosphatidylinositol species that may be shunted to the synthesis of saturated and mono-unsaturated TG, further corroborating fattyladen hepatocyte formation [118].

\section{GCKR: a possible link between glucose homeostasis and fatty liver}

Alongside PNPLA3, TM6SF2 and MBOAT7 variations, even the common loss-of-function rs1260326 C > $\mathrm{T}$ variant in the GCKR gene, encoding the p.P446L substitution has been widely associated with increased fasting TG concentrations, large VLDL, steatosis and liver damage [115, 119-121]. GCKR gene codifies for the glucokinase regulatory protein, that exerts a crucial role in glucose homeostasis, whereby regulating glucose influx into the hepatocytes and the activation of de novo lipogenesis. In particular, the alteration of GCKR affects glucokinase partitioning between the cytosol and nucleus, thus impairing its negative modulation in response to fructose-6-phosphate and in turn, it constitutively activates the hepatic glucose uptake [122]. On one hand, this effect may ameliorate circulating fasting glucose and insulin levels, on the other it may provide malonyl-CoA as substrate for de novo lipogenesis and it may avoid fatty acid oxidation through the inhibition of carnitine-palmitoyltransferase, favoring glycolysis and steatosis development [123, 124]. Santoro et al. [125], reported for the first time the rate of de novo lipogenesis through incorporation of deuterium into the palmitate contained in the VLDL after the administration of a carbohydrate drink (75 g glucose and $25 \mathrm{~g}$ fructose) in obese adolescents. These authors demonstrated that the GCKR rs1260326 variation in homozygosity increased hepatic lipid synthesis in obese adolescents, as a result of the enhanced glycolytic carbon flux to TG formation [125]. This study was the first that revealed in pediatric subjects that a common variant might favor steatosis onset by enhancing the ability of the liver to convert carbohydrate into TG [125]. Moreover, it has been demonstrated that the combination of PNPLA3 and GCKR minor alleles may explain up to $32 \%$ of the liver fat content in Caucasian obese children, 39\% in African-Americans and $15 \%$ in Hispanics [120]. The joint effect of GCKR variant with PNPLA3 has been indicated even by the strong increased risk of MAFLD in histologically confirmed MAFLD patients [126]. Furthermore, this variant has been also associated with increased susceptibility to NASH and NASH-derived HCC [126, 127] and enhanced fibrosis in adult MAFLD patients along with elevated serum TG levels, without altering LDL and HDL cholesterol levels and cardiovascular risk $[119,128]$.

\section{Protective inheritable factors against liver injuries: the case of hydroxysteroid 17-beta dehydrogenase 13 (HSD17B13) and protein phosphatase 1 regulatory subunit 3B (PPP1R3B) variations}

In 2018, an exome-wide sequencing identified the rs72613567 variant, which has been shown to protect against histological steatohepatitis and against clinically significant fibrosis and cirrhosis, in both MAFLD and ALD [129]. The rs72613567 is an insertion of an adenine adjacent to the donor splice site of the last exon (TA allele), resulting in a truncated transcript, reduced expression and impaired enzymatic activity of the HSD17B13, which is widely expressed in the hepatocytes, at the lipid droplets surface [129-131]. The precise function of HSD17B13 is currently under definition. However, it has been demonstrated that HSD17B13 is up-regulated in MAFLD-affected patients, at the lipid droplets surface and in experimental models of NASH [131]. In particular, HSD17B13 over-expression is enabled to exacerbate the number and the size of the lipid droplets in cultured hepatocytes [131]. Conversely, HSD17B13 KO mice had an impairment in hepatic-lipid metabolism, resulting in an increased TG content into the liver and steatosis onset [132], directly inducing de novo lipogenesis through SREBP1 and fatty acid synthase over-expression [131], without 
altering reproductive performance and serum steroid concentrations [132]. Thus, the effect of HSD17B13 on hepatic steatosis remains controversial. The rs72613567 variant has never been related to a reduced risk of hepatic steatosis development [129]. Moreover, Kozlitina et al. [133], revealed also the presence of another loss of function variant in HSD17B13 (c.573delC, rs143404524) more common in African-Americans than in Hispanics or Caucasians, that seems to confer a protection against chronic liver disease, but the association between this variant and hepatic TG content has not been found. In a case-control study, Pirola et al. [134], reported that TA allele protects against histological steatohepatitis, ballooning degeneration, lobular inflammation and fibrosis in MAFLD patients. This data is even more confirmed in a very recent GWAS that demonstrated that the HSD17B13 protective effect is more relevant to development of steatohepatitis than progression of fibrosis [115]. Furthermore, the rs72613567 variant has been related to a reduced risk of elevated transaminases and HCC in 111, 612 individuals from the Danish general population and in 3, 315 European patients, respectively $[135,136]$. The likely mechanism behind these genetic associations seems to be due to an increased concentration of phospholipids in the liver of carriers compared to noncarriers, that is coupled to a down-regulation of pro-inflammatory genes [137].

An interaction between HSD17B13 rs72613567 and PNPLA3 I148M has been reported. Indeed, the HSD17B13 TA allele seems to be able to mitigate the effect of PNPLA3 I148M variant on liver injuries, without affecting hepatic fat accumulation [129]. Furthermore, the lowering effect of HSD17B13 variant on transaminases is amplified in patients with high risk of fatty liver, such as in carriers of the PNPLA3 variant [135]. All these notions introduce the concept that the modulation of HSD17B13 in patients carrying PNPLA3 G allele may represent a potential therapeutic strategy against chronic liver diseases. However, the precise mechanism linking the HSD17B13 variant with inflammation and fibrosis remains uncharacterized.

Another variant that has been shown to be protective against liver damage is the rs4841132 $\mathrm{G}>\mathrm{A}$, which enhances the expression of PPP1R3B, involved in glycogen synthesis $[121,138]$. Indeed, it has been reported to reduce the risk of MAFLD but at the same time may favor liver disease by facilitating glycogen accumulation $[138,139]$. In addition, the minor $A$ allele associated with increased aminotransferases and liver disease diagnosis [138]. Conversely, in a rodent model, hepatic genetic deletion of $P P P 1 R 3 B$ significantly reduce glycogen synthase protein abundance, glucose incorporation into hepatic glycogen, total hepatic glycogen content and fasting plasma glucose compared to wild-type littermates [140]. Therefore, the overall impact of $P P P 1 R 3 B$ variation on hepatic fat and progressive liver damage remains disputed. However, Dongiovanni et al. [139], demonstrated that $P P P 1 R 3 B$ variant was associated with protection from steatosis and fibrosis, resulting into protection from HCC development in individuals at high risk of MAFLD, but not in the general population.

\section{Genetic landscape in MAFLD pathophysiology}

Several other less frequent variants may influence MAFLD pathophysiology and progression, by exerting an effect on a particular aspect of the disease. Overwhelming data demonstrated that IR is a key player in the development of MAFLD and progressive forms [141, 142]. Indeed, IR correlates with the severity of liver fibrosis [141], the main determinant of MAFLD prognosis [143], and fibrosis progression is observed in T2D patients with MAFLD, irrespective of NASH $[144,145]$. Thus, genetic variants that impair insulin receptor (InsR) signaling activation may favor fibrosis development in MAFLD [146]. For instance, the loss-of-function rs1801278 (G972R) mutation in insulin receptor substrate (IRS1) and the gain-of-function mutation in the ectonucleotide pyrophosphatase/phosphodiesterase1 (ENPP1) 121Q genes are both associated with a marker reduction of insulin sensitivity, increased body weight, dyslipidemia and liver disease severity, in particular in terms of fibrosis [146]. Conversely, the rs2954021 polymorphism in the tribbles homolog1 (TRIB1), involved in glycogenesis, impacts on MAFLD onset and circulating TG [147].

Several mutations in genes governing hepatic lipid secretion and utilization have also been shown to be causative of inherited fatty liver. Indeed, variants within Apolipoprotein B ( $A P O B$ ), involved in VLDL assembly and release, have been associated with low circulating lipoproteins and in turn, severe hepatic fat deposition, that may favor the progression of liver injury until HCC $[148,149]$. Similar findings have been observed even 
in presence of microsomal triglyceride transfer protein (MTTP) variations that cause VLDL retention [150]. Another component of VLDL particles, chylomicrons, and HDL cholesterol is the apolipoprotein C3 (APOC3). Two common promoter variants (APOC3 T-455C and C-482T) predispose to liver fat accumulation in Indian individuals, but not in other ethnic groups, suggesting that genetic factors influencing TG metabolism outside of the liver are less involved in the pathogenesis of progressive MAFLD [151-153].

More recently, Dongiovanni et al. [154], demonstrated that the proprotein convertase subtilisin/kexin type 7 (PCSK7) rs236918 G > C variant impacts on circulating lipids and liver damage in a large cohort of MAFLD patients, bridging atherogenic dyslipidemia with hepatic inflammation and fibrosis. In this cohort, the variant did not impact on hepatic fat accumulation overall, however stratifying patients for the presence of the PNPLA3 I148M allele, the rs236918 polymorphism seems to be also associated with more severe steatosis. In particular, in HepG2 cells carrying the PNPLA3 I148M allele in homozygosity, PCSK7 genetic deletion reduced lipogenesis, fat accumulation, inflammation and fibrogenesis, even after the exposure to a FFA challenge [154]. In addition, Huang et al. [155], identified a significant correlation between the minor C allele and a strong increase in fasting insulin levels and homeostatic model assessment for IR in response to high-carbohydrate diet consumption.

Furthermore, alterations in PCSK9, another member of the proprotein convertase subtilisin/kexin family, have been broadly associated with familial hypercholesterolemia [156], severe steatosis [157] and cardiovascular risk [158], as a consequence of its role in the modulation of low-density lipoprotein LDL uptake. PCSK9 expression is strongly influenced by nutritional status and its expression impressively decreases in mice after $24 \mathrm{~h}$ of fasting. On the contrary, PCSK9 mRNA levels are restored trough SREBP1c activation upon high carbohydrate refeeding or insulin stimulation [159]. Loss-of-function mutations in PCSK9 have been reported to reduce plasma LDL cholesterol, without altering hepatic fat content [160].

Another rare familiar cause of hepatic disorders is represented by the alterations of lysosomal acid lipase (LIPA) gene, that cause a lysosomal acid lipase (LAL) deficiency. LAL is responsible for the hydrolysis of cholesteryl esters, TG, and LDL particles into free cholesterol and fatty acids. Thus, its functional impairment induces the accumulation of these un-hydrolyzed compounds in hepatocytes, whereby favoring hypercholesterolemia, cardiovascular disease, hepatic steatosis and cirrhosis [161,162].

Furthermore, even variants implicated in fatty acid uptake and metabolism, such as the rs56225452 in fatty acid transport proteins (FATP5) or the rs13412852 in Lipin1 (LPIN1), may influence IR, steatosis and ALT levels, by regulating the flux of FFAs to the liver $[163,164]$.

\section{Genetic variants associated with progressive liver damage}

In the contest of fatty liver, a broad number of adverse events may precipitate liver injury towards end-stage conditions. Indeed, oxidative stress, ER and mitochondrial dysfunctions, innate immune inflammation [165], intestinal high permeability, dysbiosis and enhanced circulating gut-derived by-products [166], and HSCs activation [167] participate to the switching from simple steatosis to NASH and fibrosis.

The stronger association reported for genetic variants involved in the regulation of inflammatory response is provided by tumor necrosis factor $\alpha(T N F-\alpha)$ and interleukin 28 (IL28) polymorphisms. The latter gene codifies for the interferon $\lambda 3 / \lambda 4$ (IFNL3/4), and the rs12979860 CC variant has been widely related to elevated production of interferon $\lambda 3$, but not to interferon $\lambda 4$ [168]. Thus, interferon $\lambda 3$ is responsible for the increased clearance of hepatitis $\mathrm{C}$ virus, and more severe NASH and fibrosis in MAFLD patients [169, 170]. In particular, it has been proposed, by Eslam et al. [171], a gene model, referred to as FibroGENE, based on the rs12979860 genotype, age, gender and the routinely assessed clinical and laboratory variables to predict significant fibrosis. The rs 12979860 is in high linkage disequilibrium with the rs368234815 TT > $\delta G$ in IFNL4. Carriers of the rs368234815 TT allele are characterized by more severe necroinflammation and fibrosis compared to non-carriers [172]. Opposite results have been observed in presence of the rs3480 A>G polymorphism in the fibronectin type III domain-containing protein 5 (FNDC5) gene, that encode irisin, a novel myokine, that mediated fibrogenic activation and collagen synthesis in HSCs [173, 174]. On the contrary, it has been described that the minor $\mathrm{G}$ allele is associated with more severe steatosis, likely by modulating 
irisin expression in MAFLD patients [174]. As well as, the rs2228603 variant in Neurocan, the rs12137855 SNP in lysophospholipase-like 1 (LYPLAL1), and the rs10883437 near the carboxypeptidase $\mathrm{n}$ subunit 1 have related to advanced liver damage in MAFLD subjects $[175,176]$.

Even more, several line of evidence indicates that the gut-derived fibroblast growth factor (FGF) 19 signal, mainly involved in the regulation of lipid and carbohydrate metabolism in response to nutritional status, is associated with metabolic diseases and MAFLD pathogenesis [177]. Dongiovanni et al. [178], recently reported that the rs17618244 $\mathrm{G}>\mathrm{A}$ variant in the $\beta$-Klotho $(K L B)$ gene, encoding the hepatic co-receptor of the FGF19 receptor (fibroblast growth factor receptor 4, FGFR4), leads to reduced KLB plasma levels, severe lobular inflammation, ballooning and fibrosis in obese MAFLD pediatric patients. Indeed, KLB reduced expression causes the over-expression of genes involved in lipotoxicity and inflammation [178]. Thus, therapeutic strategies aimed to ameliorate KLB levels may represent an attractive approach to MAFLD management.

Furthermore, the rs4374383 non-coding variant in the macrophage c-mer tyrosine kinase (MERTK) has been associated with a protection against fibrosis in both MAFLD and in viral hepatitis C, thereby downmodulating the expression of MERTK, a tyrosine kinase that initiates the removal of dying cells by phagocytes and that is involved in the activation of HSCs $[179,180]$. According to these notions, it has been recently reported that MerTK cleavage in hepatic macrophages is reduced during the transition from simple steatosis to NASH, promoting in turn transforming growth factor $\beta$ (TGF- $\beta$ ) release and HSCs activation [181].

During their proliferation and activation, the HSCs express Krueppel-like factor 6 (KLF6). Alternative splicing of the KLF6 gene associates with mild MAFLD and reduced fibrosis [182]. In addition, variants that predispose to hepatic iron depot formation, such as alterations in HFE and TMPRSS6 genes, are also correlated with more advance fibrosis in MAFLD patients [183].

Finally, the susceptibility to fibrogenesis and carcinogenesis is also influenced by cellular senescence and cell cycle arrest. Therefore, the rs762623 in cyclin dependent kinase inhibitor 1A (CDKI1A) which encodes the cellular senescence marker p21, was significantly associated with disease progression in MAFLD [184]. Likewise, telomerase reverse transcriptase (TERT) gene loss-of-function mutations associate with familial cirrhosis and accelerate HCC development [185].

\section{Recent advances in mitochondrial dysfunctions: a new step to fill the gap between fatty liver and steatohepatitis}

Mitochondrial dysfunction is closely related to the pathogenesis of MAFLD so much so that in the past years the latter has been defined as a mitochondrial disease [186]. In the early stages of MAFLD, mitochondrial activity increases in response to hepatic IR and FFA accumulation. Sustained mitochondrial oxidative flux results in increased reactive oxygen species production associated with mitochondrial DNA damage, ER stress, tissue inflammation and cell death which may contribute to the progression to NASH and more advanced liver damage [187].

Common polymorphisms in genes regulating mitochondrial function have been associated with MAFLD development and to its progressive forms. The superoxide dismutase 2 (SOD2) gene encodes the antioxidant enzyme manganese superoxide dismutase. The rs4880 C47T polymorphism in the SOD2 gene results in a valine to alanine amino acid substitution in the signal region targeting the protein to the mitochondrial matrix, where it exerts its function and the T allele has been related to higher enzyme activity. Al-Serri et al. [188], by exploiting both case-control and intra-familial association studies found a consistent association between the rs4880 and advanced fibrosis in MAFLD patients providing a convincing evidence that mitochondria-derived oxidative stress is crucial for the development of fibrosing NASH. In keeping with these findings, Namikawa et al. [189], reported that 63 patients with biopsy-proven NASH had a higher incidence of the SOD2 T/T genotype compared to 150 healthy controls

The uncoupling protein 2 (UCP2) is involved in the regulation of mitochondrial lipid efflux and oxidative metabolism and its hepatic expression has been found to be increased in NASH patients leading to a proton leak and to a reduced redox pressure on the mitochondrial respiratory chain, thus exerting a protective role 
on liver damage progression [190]. Fares et al. [191], performed a case-control study in which 688 Italian patients with biopsy-proven NASH and 232 healthy controls were enrolled to investigate the association between the $-866 \mathrm{G}>\mathrm{A} U C P 2$ promoter region polymorphism and the susceptibility to develop NASH. The authors found that homozygosity for the -866A UCP2 allele was protective towards NASH and was associated with higher hepatic UCP2 expression.

The uncoupling protein 3 encoded by the UCP3 gene is a mitochondrial proton carrier which uncouples the oxidative phosphorylation by increasing the proton leak of the inner mitochondrial membrane. The UCP3 transporter is considered to be protective against oxidative stress derived from-oxidation of fatty acids. Aller et al. [192], demonstrated that a non-coding variant in the promoter $(-55 \mathrm{C}>\mathrm{T}$, rs1800849) of UCP3 was associated with IR, low adiponectin levels, moderate-severe steatosis and inflammation in obese patients with MAFLD.

Sirtuins (SIRTs) which are a family of nicotinamide adenine dinucleotide (NAD ${ }^{+}$)-dependent deacetylases involved in cellular metabolism and UCPs may modify oxidative stress thereby affecting the risk of atherosclerosis and cardiovascular diseases. In a group of 1, 018 stroke-free subjects from the Northern Manhattan Study (NOMAS) who had high-definition carotid ultrasonography and a GWAS data available, the SIRT6 rs107251 and the SIRT5 rs12216101 were associated with an increased risk for carotid plaque, whereas T-carriers of the UCP5 SNP rs5977238 had a decreased risk [193]. However, the impact of genetic variations in SIRTs genes has not been deeply investigated in MAFLD patients who are highly susceptible to cardiovascular complication.

It's only recently been described a novel common missense variant (rs2642438 A165T) in the mitochondrial amidoxime-reducing component 1 (MARC1) gene which encodes for the mitochondrial amidoxime reducing component 1 , a molybdenum-containing enzyme. The $\mathrm{A} 165 \mathrm{~T}$ variant is located at the $\mathrm{N}$-terminal domain which anchors the protein to the outer membrane of the mitochondria. The threonine to alanine amino acid substitution results in a truncating protein making the rs2642438 variant a loss of function mutation. The A165T variant has been associated with protection against all-cause cirrhosis, reduced hepatic fat content and lower levels of liver enzymes pointing to MARC1 as a potential therapeutic target for liver diseases although further studies are required to clarify its function and its role in oxidative stress regulation [194]. A schematic over-view of the main genetic risk factors involved in MAFLD onset and progression is represented in Figure 1 and in Table 1.

\section{Polygenic risk scores (PRSs): clinical translation of genetics}

In the past years, single candidate gene studies and GWAS have contributed to pinpoint the role of genetic variants in MAFLD pathogenesis and progression. Similarly, to what has been done for other complex disorders, a recent approach is to combine individual loci into PRSs to convert genetic data into a measure of the risk of developing MAFLD. The aggregation of these scores with the most common risk factors may be helpful in identifying those patients at higher risk to develop more severe liver damage and to decide the more effective therapeutic strategy [195].

We firstly applied a mendelian randomization analysis and a PRS to demonstrate that hepatic steatosis promotes the full spectrum of liver disease and the impact of risk alleles in PNPLA3, TM6SF2, MBOAT7 and GCKR on liver damage is proportional to their effect on hepatic fat accumulation [28]. In keeping with these findings, Di Costanzo et al. [196], evaluated the impact of genetic and metabolic variables on liver fat accumulation in a cohort of obese children. They found that genetic variants contribute more strongly to fatty liver compared to IR which is a known driver of steatosis and fibrosis. Moreover, hepatic fat content variation was explained for $8.7 \%$ by metabolic factors and for $16.1 \%$ by the weighted effect of PNPLA3, TM6SF2, and GCKR variants [196]. Similarly, in a cohort of 2, 042 children Suomela et al. [197], demonstrated that a combined score including BMI, insulin levels and genetic variants in PNPLA3 and TM6SF2 genes was more accurate to predict fatty liver compared to the one used in adulthood based only on BMI and insulin. 


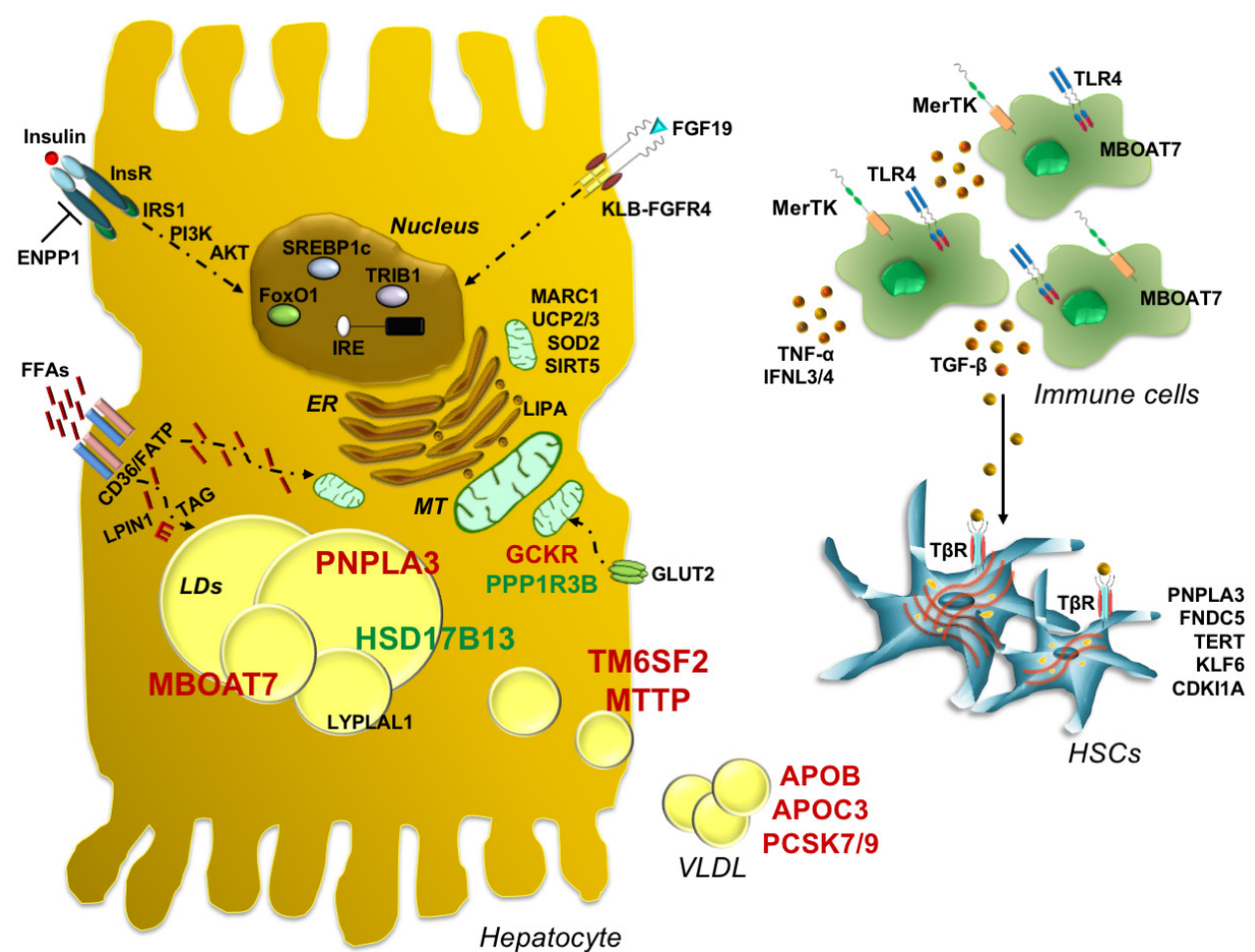

Figure 1. Genetic contribution to MAFLD pathogenesis and progression towards worsen stages. Schematic representation of the main genetic risk variants implicated in MAFLD onset and progression, which highlights their possible functional effects. PNPLA3 is an intracellular membrane lipase, localized on the surface of lipid droplets in hepatocytes where catalyzes TG hydrolysis. The PNPLA3 148M variant increases hepatic TG content upon accumulation of the mutant protein on the surface of lipid droplets, reducing TG turnover and dismissal. The I148M variant impairs the amount of released VLDL, exacerbating fat deposition. TM6SF2 is directly involved in VLDL secretion, whereas MBOAT7 catalyzes the transfers of PUFA, such as arachidonoyl-CoA to lysophospholipids, thus maintaining the fluidity of membranes. Thus, the TM6SF2 E167K variant impairs physiological VLDL secretion and affects cholesterol metabolism and TG synthesis, while the rs 641738 variant in MBOAT7 reduces membrane fluidity and dynamism by altering phospholipid acyl-chains remodeling and lipogenic program in hepatocytes, while in immune cells it enhances the amount of free arachidonic acid, thus triggering inflammation. On the contrary genetic variants such as $H S D 17 B 13$ and $P P P 1 R 3 B$ may exert a protective effect against liver injuries. Furthermore, inherited variations that influence glucose and insulin signaling, FFA uptake, fat deposition and VLDL assembly and release may contribute to fatty liver development. Viceversa, hyperinsulinemia and elevated FFAs derived from adipose tissue exacerbate fat deposition induced by genetic modifiers, even activating de novo lipogenesis, through SREBP-1c. Common polymorphisms in genes regulating mitochondrial (MT) function have been associated with MAFLD development and to its progressive forms, further corroborating the switching from simple steatosis to NASH and fibrosis. In addition, it has been reported that genetic variants involved in the regulation of inflammatory response in immune cells and in the activation of HSCs to myofibroblasts phenotype may precipitate fatty liver to worsen conditions. Finally, the combined effect of PNPLA3, TM6SF2, and MBOAT7 genetic variants may predispose to an increased risk of progressive liver damage. PI3K: Phosphoinositide 3-kinase; FOXO1: Forkhead box protein O1; CD36: cluster of differentiation 36; TLR4: toll like receptor 4

Krawczyk et al. [106], analyzed the joint effect of PNPLA3 I148M, TM6SF2 E167K, and MBOAT7 rs641738 variants on liver damage in a large cohort of patients with fatty liver and demonstrated that increasing number of risk alleles was associated with higher aminotransferase, which may reflect hepatic injury in MAFLD. The additive effect of PNPLA3 and TM6SF2 gene variation seems to affect lipid metabolism and MAFLD possibly by upregulating the expression of genes involved in de novo lipogenesis [198]. Risk estimation of progressive MAFLD by PRS was also calculated in a Japanese study which demonstrated that the effect of the risk alleles, namely PNPLA3, GATAD2A and GCKR was cumulative and increased the risk of NASH [127].

Genetic variants predisposing to hepatic fat accumulation promote HCC development and may be useful biomarkers for patients' stratification. We previously demonstrated that the number of genetic risk variants in PNPLA3, TM6SF2 and MBOAT7 was strongly associated with HCC, with a 13.4-fold higher risk in MAFLD patients carrying five risk alleles as compared to none [103]. Gellert-Kristensen et al. [199], have recently assessed that a PRS including the genetic variant in PNPLA3, TM6SF2 and HSD17B13 genes is associated with a 12 -fold and 29-fold higher risk to develop cirrhosis and HCC respectively, in the general population.

All these studies raise the important question about the possibility to use these scores in the clinical setting to predict the development of MAFLD and its progression to more severe forms and how to combine them with the metabolic risk factors to apply the appropriate therapeutic intervention. The new challenge is 
to understand how to use the genetic architecture of MAFLD, which has been explored in the past years, to identify new druggable targets and to accelerate the MAFLD drug discovery pipeline [200].

Table 1. List of the main genetic variants associated with the histological spectrum of MAFLD

\begin{tabular}{|c|c|c|c|c|c|}
\hline Variant & Gene & Function & Effect & Impact & Phenotype \\
\hline rs738409 C > G & PNPLA3 & Lipid remodeling & p.I148M & Loss-of-function & $\begin{array}{l}\uparrow \mathrm{MAFLD}, \mathrm{NASH}, \\
\text { fibrosis, HCC }\end{array}$ \\
\hline rs58542926 C > T & TM6SF2 & VLDL secretion & p.E167K & Loss-of-function & $\uparrow$ MAFLD, NASH, fibrosis \\
\hline rs641738 C > T & TMC4/MBOAT7 & Lipid remodeling & p.G17E & Loss-of-function & $\begin{array}{l}\uparrow \text { MAFLD, NASH, } \\
\text { fibrosis, HCC }\end{array}$ \\
\hline rs1260326 C > T & GCKR & $\begin{array}{l}\text { Regulation of de novo } \\
\text { lipogenesis }\end{array}$ & p.P446L & Loss-of-function & $\uparrow$ MAFLD, NASH, fibrosis \\
\hline rs72613567 T > TA & HSD17B13 & Lipid remodeling & Truncated protein & Loss-of-function & $\downarrow \mathrm{NASH}$, fibrosis, HCC \\
\hline rs4841132 G > A & $P P P 1 R 3 B$ & Glycogen synthesis & Non-coding & Gain-of-function & $\downarrow$ MAFLD, fibrosis, HCC \\
\hline rs1801278 A > C & IRS1 & Insulin signaling & p.G972R & Loss-of-function & $\uparrow$ Fibrosis \\
\hline rs1044498 A > C & ENPP1 & Insulin signaling & p.K121Q & Gain-of-function & $\uparrow$ Fibrosis \\
\hline rs2954021 G > A & TRIB1 & $\begin{array}{l}\text { Regulation of de novo } \\
\text { lipogenesis }\end{array}$ & Non-coding & Gain-of-function & $\uparrow$ MAFLD \\
\hline rs12137855 C > T & LYPLAL1 & Lipid metabolism & Intronic & Loss-of-function & $\uparrow$ MAFLD \\
\hline Several & $A P O B$ & VLDL secretion & Protein change & Loss-of-function & $\begin{array}{l}\uparrow \text { MAFLD, NASH, } \\
\text { fibrosis, HCC }\end{array}$ \\
\hline Several & MTTP & VLDL secretion & Protein change & Loss-of-function & $\uparrow$ MAFLD \\
\hline rs236918 G > C & PCSK7 & $\begin{array}{l}\text { Membrane transferrin } \\
\text { receptor shedding } \\
\text { and regulation of } \\
\text { circulating lipids }\end{array}$ & Intronic & Gain-of-function & $\uparrow \mathrm{NASH}$, fibrosis \\
\hline Several & PCSK9 & LDL uptake & Protein change & Loss-of-function & $\begin{array}{l}\text { No evidence of } \\
\text { association with steatosis }\end{array}$ \\
\hline Several & LIPA & Lipid remodeling & Protein change & LAL deficiency & $\uparrow$ MAFLD, NASH, fibrosis \\
\hline rs56225452 G > A & FATP5 & FFAs uptake & Non-coding & Gain-of-function & $\uparrow \mathrm{NASH}$, fibrosis \\
\hline rs13412852 C > T & LPIN1 & Lipid metabolism & Intronic & NA & $\downarrow \mathrm{NASH}$, fibrosis \\
\hline rs17618244 G > A & $K L B$ & $\begin{array}{l}\text { FGF19/FGFR4 } \\
\text { pathway }\end{array}$ & $\mathrm{R} 728 \mathrm{Q}$ & Loss-of-function & $\downarrow \mathrm{NASH}$, fibrosis \\
\hline rs4374383 G > A & MERTK & Innate immunity & Intronic & Loss-of-function & $\downarrow$ Fibrosis \\
\hline rs3750861 G > A & $K L F 6$ & HSCs activation & $\begin{array}{l}\text { Splice variant } \\
\text { IVS1-27G }\end{array}$ & Loss-of-function & $\downarrow$ Fibrosis \\
\hline Several & TERT & $\begin{array}{l}\text { Telomere } \\
\text { maintenance }\end{array}$ & Protein change & Loss-of-function & $\uparrow$ Fibrosis, HCC \\
\hline rs12979860 C > T & IL28B & Innate immunity & $\begin{array}{l}\text { Alternative } \\
\text { IFNL3/4 } \\
\text { transcription }\end{array}$ & Loss-of-function & $\downarrow$ NASH, Fibrosis \\
\hline $\mathrm{rs} 3480 \mathrm{~A}>\mathrm{G}$ & FNDC5 & HSCs activation & Non-coding & Loss-of-function & $\downarrow$ Fibrosis \\
\hline rs4880 C > T & SOD2 & $\begin{array}{l}\text { Mitochondrial } \\
\text { antioxidant }\end{array}$ & p.A16V & Loss-of-function & $\uparrow$ Fibrosis \\
\hline rs695366 G > A & UCP2 & $\begin{array}{l}\text { Mitochondrial lipid } \\
\text { metabolism Oxphos }\end{array}$ & $\begin{array}{l}-866 \text { promoter } \\
\text { variant }\end{array}$ & Gain-of-function & $\downarrow \mathrm{NASH}$, fibrosis \\
\hline rs2642438 G > A & MARC1 & $\begin{array}{l}\text { Mitochondrial } \\
\text { detoxification }\end{array}$ & A165T & Loss-of-function & $\downarrow$ MAFLD, NASH, fibrosis \\
\hline
\end{tabular}

NA: not applicable

\section{Concluding remarks}

Genetics plays a crucial role in the development of MAFLD and in its progression towards NASH and HCC. Inherited variant in PNPLA3, TM6SF2 and MBOAT7 genes provide the strongest evidence for association with MAFLD but it remains to be determined how genetic data may be translated in the clinical setting. 
Both PNPLA3 and TM6SF2 have been related with "metabolically silent" MAFLD thus contributing to its development and progression even in the absence of metabolic factors. Moreover, genetically determined fat accumulation is associated with biochemical markers of liver damage and higher risk of fibrosis. The opportunity to aggregate genetic variants in polygenic risk scores and to combine them with metabolic information represents an appealing therapeutic choice to design preventive intervention in higher risk individuals with MAFLD.

\section{Abbreviations}

ALD: alcoholic liver disease

ALT: alanine aminotransferase

APOB: Apolipoprotein B

APOC3: apolipoprotein C3

ATGL: adipose triglyceride lipase

CGI-58: comparative gene identification-58

DAGs: diacylglycerols

ENPP1: ectonucleotide pyrophosphatase/phosphodiesterase1

ER: endothelial reticulum

FATP5: fatty acid transport proteins

FFAs: free fatty acids

FGF: fibroblast growth factor

FGFR4: fibroblast growth factor receptor 4

FNDC5: fibronectin type III domain-containing protein 5

GCKR: Glucokinase regulator

GWAS: genome-wide association study

HCC: hepatocellular carcinoma

HSCs: hepatic stellate cells

HSD17B13: hydroxysteroid 17-beta dehydrogenase 13

IFNL3/4: interferon $\lambda 3 / \lambda 4$

IL28: interleukin 28

InsR: impair insulin receptor

IR: insulin resistance

IRS1: insulin receptor substrate

KLB: $\beta$-Klotho

KLF6: Krueppel-like factor 6

KO: knock-out

LAL: lysosomal acid lipase

LDL: low-density lipoprotein

LIPA: lysosomal acid lipase

LYPLAL1: lysophospholipase-like 1

MAFLD: metabolic associated fatty liver disease

MARC1: amidoxime-reducing component 1

MBOAT7: membrane bound o-acyltransferase domain-containing 7

MERTK: macrophage c-mer tyrosine kinase 
MetS: metabolic syndrome

MRI-PDFF: magnetic resonance imaging proton-density fat fraction

MTTP: microsomal triglyceride transfer protein

NASH: nonalcoholic steatohepatitis

PCs: phosphatidylcholines

PCSK7: proprotein convertase subtilisin/kexin type 7

PNPLA2: patatin-like phospholipase domain-containing 2

PNPLA3: patatin-like phospholipase domain-containing 3

PPP1R3B: protein phosphatase 1 regulatory subunit 3B

PRSs: polygenic risk scores

PUFAs: polyunsaturated fatty acids

SIRTs: sirtuins

SNP: single nucleotide polymorphism

SOD2: superoxide dismutase 2

SREBP1c: sterol regulatory element-binding protein 1

T2D: type 2 diabetes

TERT: telomerase reverse transcriptase

TG: triglyceride

TGF- $\beta$ : transforming growth factor $\beta$

TM6SF2: transmembrane 6 superfamily member 2

TMC4: transmembrane channel like 4

$T N F-\alpha$ : tumor necrosis factor $\alpha$

TRIB: tribbles homolog1

UCP2: uncoupling protein 2

VLDL: very-low density lipoproteins

\section{Declarations}

\section{Author contributions}

MM, ML and PD all took part in writing the manuscript, preparing figures and reading and approving the final draft. All authors have read and agreed to the published version of the manuscript.

\section{Conflicts of interest}

The authors declare that they have no conflicts of interest.

\section{Ethical approval}

Not applicable.

\section{Consent to participate}

Not applicable.

\section{Consent to publication}

Not applicable.

Availability of data and materials

Not applicable. 


\section{Funding}

The study was supported by the Ricerca Corrente Fondazione IRCCS Cà Granda and Ricerca Finalizzata Ministero della Salute RF-2013-02358319. The funders had no role in study design, data collection and analysis, decision to publish, or preparation of the manuscript.

\section{Copyright}

(C) The Author(s) 2020.

\section{References}

1. Younossi ZM, Koenig AB, Abdelatif D, Fazel Y, Henry L, Wymer M. Global epidemiology of nonalcoholic fatty liver disease-Meta-analytic assessment of prevalence, incidence, and outcomes. Hepatology. 2016;64:73-84.

2. Eslam M, Sanyal AJ, George J. MAFLD: a consensus-driven proposed nomenclature for metabolic associated fatty liver disease. Gastroenterology. 2020;158:1999-2014.e1.

3. Eslam M, Newsome PN, Sarin SK, Anstee QM, Targher G, Romero-Gomez M, et al. A new definition for metabolic dysfunction-associated fatty liver disease: an international expert consensus statement. J Hepatol. 2020;73:202-9.

4. Cholankeril G, Wong RJ, Hu M, Perumpail RB, Yoo ER, Puri P, et al. Liver transplantation for nonalcoholic steatohepatitis in the US: temporal trends and outcomes. Dig Dis Sci. 2017;62:2915-22.

5. Younossi ZM, Henry L, Bush H, Mishra A. Clinical and economic burden of nonalcoholic fatty liver disease and nonalcoholic steatohepatitis. Clin Liver Dis. 2018;22:1-10.

6. Day CP. From fat to inflammation. Gastroenterology. 2006;130:207-10.

7. Wong RJ, Aguilar M, Cheung R, Perumpail RB, Harrison SA, Younossi ZM, et al. Nonalcoholic steatohepatitis is the second leading etiology of liver disease among adults awaiting liver transplantation in the United States. Gastroenterology. 2015;148:547-55.

8. Marchesini G, Brizi M, Bianchi G, Tomassetti S, Bugianesi E, Lenzi M, et al. Nonalcoholic fatty liver disease: a feature of the metabolic syndrome. Diabetes. 2001;50:1844-50.

9. Byrne CD, Targher G. NAFLD: a multisystem disease. J Hepatol. 2015;62:S47-64.

10. Dongiovanni P, Valenti L. A nutrigenomic approach to non-alcoholic fatty liver disease. Int J Mol Sci. 2017;18:1534.

11. Meroni M, Longo M, Rustichelli A, Dongiovanni P. Nutrition and genetics in NAFLD: the perfect binomium. Int J Mol Sci. 2020;21:2986.

12. Dongiovanni P, Valenti L. Genetics of nonalcoholic fatty liver disease. Metabolism. 2016;65:1026-37.

13. Meroni M, Longo M, Erconi V, Valenti L, Gatti S, Fracanzani AL, et al. mir-101-3p downregulation promotes fibrogenesis by facilitating hepatic stellate cell transdifferentiation during insulin resistance. Nutrients. 2019;11:2597.

14. Dongiovanni P, Meroni M, Longo M, Fargion S, Fracanzani AL. miRNA signature in NAFLD: a turning point for a non-invasive diagnosis. Int J Mol Sci. 2018;19:3966.

15. Meroni M, Longo M, Dongiovanni P. Alcohol or gut microbiota: who is the guilty? Int J Mol Sci. 2019;20:4568.

16. Meroni M, Longo $M$, Dongiovanni $P$. The role of probiotics in nonalcoholic fatty liver disease: a new insight into therapeutic strategies. Nutrients. 2019;11:2642.

17. Dongiovanni P, Anstee QM, Valenti L. Genetic predisposition in NAFLD and NASH: impact on severity of liver disease and response to treatment. Curr Pharm Des. 2013;19:5219-38.

18. Dongiovanni P, Romeo S, Valenti L. Genetic factors in the pathogenesis of nonalcoholic fatty liver and steatohepatitis. Biomed Res Int. 2015;2015:460190. 
19. Schwimmer JB, Celedon MA, Lavine JE, Salem R, Campbell N, Schork NJ, et al. Heritability of nonalcoholic fatty liver disease. Gastroenterology. 2009;136:1585-92.

20. Willner IR, Waters B, Patil SR, Reuben A, Morelli J, Riely CA. Ninety patients with nonalcoholic steatohepatitis: insulin resistance, familial tendency, and severity of disease. Am J Gastroenterol. 2001;96:2957-61.

21. Makkonen J, Pietilainen KH, Rissanen A, Kaprio J, Yki-Järvinen H. Genetic factors contribute to variation in serum alanine aminotransferase activity independent of obesity and alcohol: a study in monozygotic and dizygotic twins. J Hepatol. 2009;50:1035-42.

22. Loomba R, Schork N, Chen CH, Bettencourt R, Bhatt A, Ang B, et al. Heritability of hepatic fibrosis and steatosis based on a prospective twin study. Gastroenterology. 2015;149:1784-93.

23. Cui J, Chen CH, Lo MT, Schork N, Bettencourt R, Gonzalez MP, et al; For The Genetics Of Nafld In Twins Consortium. Shared genetic effects between hepatic steatosis and fibrosis: a prospective twin study. Hepatology. 2016. 64:1547-58.

24. Balakrishnan M, Kanwal F, El-Serag HB, Thrift AP. Acculturation and nonalcoholic fatty liver disease risk among hispanics of mexican origin: findings from the national health and nutrition examination survey. Clin Gastroenterol Hepatol. 2017;15:310-2.

25. Browning JD, Szczepaniak LS, Dobbins R, Nuremberg P, Horton JD, Cohen JC, et al. Prevalence of hepatic steatosis in an urban population in the United States: impact of ethnicity. Hepatology. 2004;40:1387-95.

26. Fleischman MW, Budoff M, Zeb I, Li D, Foster T. NAFLD prevalence differs among hispanic subgroups: the multi-ethnic study of atherosclerosis. World J Gastroenterol. 2014;20:4987-93.

27. Guerrero R, Vega GL, Grundy SM, Browning JD. Ethnic differences in hepatic steatosis: an insulin resistance paradox? Hepatology. 2009;49:791-801.

28. Dongiovanni P, Stender S, Pietrelli A, Mancina RM, Cespiati A, Petta S, et al. Causal relationship of hepatic fat with liver damage and insulin resistance in nonalcoholic fatty liver. J Intern Med. 2018;283:356-70.

29. Di Costanzo A, Belardinilli F, Bailetti D, Sponziello M, D’Erasmo L, Polimeni L, et al. Evaluation of polygenic determinants of non-alcoholic fatty liver disease (NAFLD) by a candidate genes resequencing strategy. Sci Rep. 2018;8:3702.

30. Krawczyk M, Bantel H, Rau M, Schattenberg JM, Grünhage F, Pathil A, et al. Could inherited predisposition drive non-obese fatty liver disease? Results from German tertiary referral centers. J Hum Genet. 2018;63:621-6.

31. Romeo S, Kozlitina J, Xing C, Pertsemlidis A, Cox D, Pennacchio LA, et al. Genetic variation in PNPLA3 confers susceptibility to nonalcoholic fatty liver disease. Nat Genet. 2008;40:1461-5.

32. Pingitore P, Dongiovanni P, Motta BM, Meroni M, Lepore SM, Mancina RM, et al. PNPLA3 overexpression results in reduction of proteins predisposing to fibrosis. Hum Mol Genet. 2016;25:5212-22.

33. Mondul A, Mancina RM, Merlo A, Dongiovanni P, Rametta R, Montalcini T, et al. PNPLA3 I148M variant influences circulating retinol in adults with nonalcoholic fatty liver disease or obesity. J Nutr. 2015;145:1687-91.

34. Huang Y, He S, Li JZ, Seo YK, Osborne TF, Cohen JC, et al. A feed-forward loop amplifies nutritional regulation of PNPLA3. Proc Natl Acad Sci U S A. 2010;107:7892-7.

35. Baulande S, Lasnier F, Lucas M, Pairault J. Adiponutrin, a transmembrane protein corresponding to a novel dietary- and obesity-linked mRNA specifically expressed in the adipose lineage. J Biol Chem. 2001;276:33336-44.

36. Stender S, Kozlitina J, Nordestgaard BG, Tybjærg-Hansen A, Hobbs HH, Cohen JC. Adiposity amplifies the genetic risk of fatty liver disease conferred by multiple loci. Nat Genet. 2017;49:842-7.

37. Santoro N, Kursawe R, D'Adamo E, Dykas DJ, Zhang CK, Bale AE, et al. A common variant in the patatin-like phospholipase 3 gene (PNPLA3) is associated with fatty liver disease in obese children and adolescents. Hepatology. 2010;52:1281-90. 
38. McGeoch LJ, Patel PR, Mann JP. PNPLA3: a determinant of response to low-fructose diet in nonalcoholic fatty liver disease. Gastroenterology. 2018;154:1207-8.

39. Fracanzani AL, Petta S, Lombardi R, Pisano G, Russello M, Consonni D, et al. Liver and cardiovascular damage in patients with lean nonalcoholic fatty liver disease, and association with visceral obesity. Clin Gastroenterol Hepatol. 2017;15:1604-11.e1.

40. Kumari M, Schoiswohl G, Chitraju C, Paar M, Cornaciu I, Rangrez AY, et al. Adiponutrin functions as a nutritionally regulated lysophosphatidic acid acyltransferase. Cell Metab. 2012;15:691-702.

41. Pingitore P, Pirazzi C, Mancina RM, Motta BM, Indiveri C, Pujia A, et al. Recombinant PNPLA3 protein shows triglyceride hydrolase activity and its I148M mutation results in loss of function. Biochim Biophys Acta. 2014;1841:574-80.

42. Dongiovanni P, Romeo S, Valenti L. Hepatocellular carcinoma in nonalcoholic fatty liver: role of environmental and genetic factors. World J Gastroenterol. 2014;20:12945-55.

43. Basantani MK, Sitnick MT, Cai L, Brenner DS, Gardner NP, Li JZ, et al. Pnpla3/Adiponutrin deficiency in mice does not contribute to fatty liver disease or metabolic syndrome. J Lipid Res. 2011;52:318-29.

44. Chen W, Chang B, Li L, Chan L. Patatin-like phospholipase domain-containing 3/adiponutrin deficiency in mice is not associated with fatty liver disease. Hepatology. 2010;52:1134-42.

45. Smagris E, BasuRay S, Li J, Huang Y, Lai KM, Gromada J, et al. Pnpla3I148M knockin mice accumulate PNPLA3 on lipid droplets and develop hepatic steatosis. Hepatology. 2015;61:108-18.

46. Lindén D, Ahnmark A, Pingitore P, Ciociola E, Ahlstedt I, Andréasson AC, et al. Pnpla3 silencing with antisense oligonucleotides ameliorates nonalcoholic steatohepatitis and fibrosis in Pnpla3 I148M knock-in mice. Mol Metab. 2019;22:49-61.

47. Wang Y, Kory N, BasuRay S, Cohen JC, Hobbs HH. PNPLA3, CGI-58, and inhibition of hepatic triglyceride hydrolysis in mice. Hepatology. 2019;69:2427-41.

48. Yang A, Mottillo EP, Mladenovic-Lucas L, Zhou L, Granneman JG. Dynamic interactions of ABHD5 with PNPLA3 regulate triacylglycerol metabolism in brown adipocytes. Nat Metab. 2019;1:560-9.

49. BasuRay S, Smagris E, Cohen JC, Hobbs HH. The PNPLA3 variant associated with fatty liver disease (I148M) accumulates on lipid droplets by evading ubiquitylation. Hepatology. 2017;66:1111-24.

50. Donati B, Motta BM, Pingitore P, Meroni M, Pietrelli A, Alisi A, et al. The rs2294918 E434K variant modulates patatin-like phospholipase domain-containing 3 expression and liver damage. Hepatology. 2016;63:787-98.

51. BasuRay S, Wang Y, Smagris E, Cohen JC, Hobbs HH. Accumulation of PNPLA3 on lipid droplets is the basis of associated hepatic steatosis. Proc Natl Acad Sci U S A. 2019;116:9521-6.

52. Negoita F, Blomdahl J, Wasserstrom S, Winberg ME, Osmark P, Larsson S, et al. PNPLA3 variant M148 causes resistance to starvation-mediated lipid droplet autophagy in human hepatocytes. J Cell Biochem. 2019;120:343-56.

53. Luukkonen PK, Nick A, Hölttä-Vuori M, Thiele C, Isokuortti E, Lallukka-Brück S, et al. Human PNPLA3I148M variant increases hepatic retention of polyunsaturated fatty acids. JCI Insight. 2019;4:e127902.

54. Franko A, Merkel D, Kovarova M, Hoene M, Jaghutriz BA, Heni M, et al. Dissociation of fatty liver and insulin resistance in I148M PNPLA3 carriers: differences in diacylglycerol (DAG) FA18:1 lipid species as a possible explanation. Nutrients. 2018;10:1314.

55. Luukkonen PK, Zhou Y, Sädevirta S, Leivonen M, Arola J, Orešič M, et al. Hepatic ceramides dissociate steatosis and insulin resistance in patients with non-alcoholic fatty liver disease. J Hepatol. 2016;64:1167-75.

56. Qadri S, Lallukka-Brück S, Luukkonen PK, Zhou Y, Gastaldelli A, Orho-Melander M, et al. The PNPLA3I148M variant increases polyunsaturated triglycerides in human adipose tissue. Liver Int. 2020; [Epub ahead of print]. 
57. Bruschi FV, Claudel T, Tardelli M, Caligiuri A, Stulnig TM, Marra F, et al. The PNPLA3 I148M variant modulates the fibrogenic phenotype of human hepatic stellate cells. Hepatology. 2017;65:1875-90.

58. Dongiovanni P, Donati B, Fares R, Lombardi R, Mancina RM, Romeo S, et al. PNPLA3 I148M polymorphism and progressive liver disease. World J Gastroenterol. 2013;19:6969-78.

59. Valenti L, Motta BM, Soardo G, Iavarone M, Donati B, Sangiovanni A, et al. PNPLA3 I148M polymorphism, clinical presentation, and survival in patients with hepatocellular carcinoma. PLoS One. 2013;8:e75982.

60. Pirazzi C, Valenti L, Motta BM, Pingitore P, Hedfalk K, Mancina RM, et al. PNPLA3 has retinyl-palmitate lipase activity in human hepatic stellate cells. Hum Mol Genet. 2014;23:4077-85.

61. Trepo E, Nahon P, Bontempi G, Valenti L, Falleti E, Nischalke HD, et al. Association between the PNPLA3 (rs738409 C>G) variant and hepatocellular carcinoma: eidence from a meta-analysis of individual participant data. Hepatology. 2014;59:2170-7.

62. Carpino G, Pastori D. PNPLA3 variant and portal/periportal histological pattern in patients with biopsyproven non-alcoholic fatty liver disease: a possible role for oxidative stress. Sci Rep. 2017;7:15756.

63. Grimaudo S, Pipitone RM, Pennisi G, Celsa C, Cammà C, Di Marco V, et al. Association between PNPLA3 rs738409 C>G variant and liver-related outcomes in patients with nonalcoholic fatty liver disease. Clin Gastroenterol Hepatol. 2020;18:935-44.e3.

64. Valenti L, Rumi M, Galmozzi E, Aghemo A, Del Menico B, De Nicola S, et al. Patatin-like phospholipase domain-containing 3 I148M polymorphism, steatosis, and liver damage in chronic hepatitis C. Hepatology. 2011;53:791-9.

65. Guyot E, Sutton A, Rufat P, Laguillier C, Mansouri A, Moreau R, et al. PNPLA3 rs738409, hepatocellular carcinoma occurrence and risk model prediction in patients with cirrhosis. J Hepatol. 2013;58:312-8.

66. Mederacke YS, Kirstein MM, Großhennig A, Marhenke S, Metzler F, Manns MP, et al. The PNPLA3 rs738409 GG genotype is associated with poorer prognosis in 239 patients with autoimmune hepatitis. Aliment Pharmacol Ther. 2020;51:1160-8.

67. Kozlitina J, Smagris E, Stender S, Nordestgaard BG, Zhou HH, Tybjaerg-Hansen A, et al. Exome-wide association study identifies a TM6SF2 variant that confers susceptibility to nonalcoholic fatty liver disease. Nat Genet. 2014;46:352-6.

68. Prill S, Caddeo A, Baselli G, Jamialahmadi O, Dongiovanni P, Rametta R, et al. The TM6SF2 E167K genetic variant induces lipid biosynthesis and reduces apolipoprotein B secretion in human hepatic 3D spheroids. Sci Rep. 2019;9:11585.

69. Smagris E, Gilyard S, BasuRay S, Cohen JC, Hobbs HH. Inactivation of Tm6sf2, a gene defective in fatty liver disease, impairs lipidation but not secretion of very low density lipoproteins. J Biol Chem. 2016;291:10659-76.

70. Fan Y, Lu H, Guo Y, Zhu T, Garcia-Barrio MT, Jiang Z, et al. Hepatic transmembrane 6 superfamily member 2 regulates cholesterol metabolism in mice. Gastroenterology. 2016;150:1208-18.

71. Ruhanen H, Nidhina Haridas PA, Eskelinen EL, Eriksson O, Olkkonen VM, Käkelä R. Depletion of TM6SF2 disturbs membrane lipid composition and dynamics in HuH7 hepatoma cells. Biochim Biophys Acta Mol Cell Biol Lipids. 2017;1862:676-85.

72. Musso G, Cassader M, Paschetta E, Gambino R. TM6SF2 may drive postprandial lipoprotein cholesterol toxicity away from the vessel walls to the liver in NAFLD. J Hepatol. 2016;64:979-81.

73. Luukkonen PK, Zhou Y, Nidhina Haridas PA, Dwivedi OP, Hyötyläinen T, Ali A, et al. Impaired hepatic lipid synthesis from polyunsaturated fatty acids in TM6SF2 E167K variant carriers with NAFLD. J Hepatol. 2017;67:128-36.

74. Li TT, Li TH, Peng J, He B, Liu LS, Wei DH, et al. TM6SF2: a novel target for plasma lipid regulation. Atherosclerosis. 2018;268:170-6. 
75. Ehrhardt N, Doche ME, Chen S, Mao HZ, Walsh MT, Bedoya C, et al. Hepatic Tm6sf2 overexpression affects cellular ApoB-trafficking, plasma lipid levels, hepatic steatosis and atherosclerosis. Hum Mol Genet. 2017;26:2719-31.

76. Mahdessian H, Taxiarchis A, Popov S, Silveira A, Franco-Cereceda A, Hamsten A, et al. TM6SF2 is a regulator of liver fat metabolism influencing triglyceride secretion and hepatic lipid droplet content. Proc Natl Acad Sci U S A. 2014;111:8913-8.

77. O'Hare EA, Yang R, Yerges-Armstrong LM, Sreenivasan U, McFarland R, Leitch CC, et al. TM6SF2 rs58542926 impacts lipid processing in liver and small intestine. Hepatology. 2017;65:1526-42.

78. Mancina RM, Sentinelli F, Incani M, Bertoccini L, Russo C, Romeo S, et al. Transmembrane-6 superfamily member 2 (TM6SF2) E167K variant increases susceptibility to hepatic steatosis in obese children. Dig Liver Dis. 2016;48:100-1.

79. Goffredo M, Caprio S, Feldstein AE, D'Adamo E, Shaw MM, Pierpont B, et al. Role of TM6SF2 rs58542926 in the pathogenesis of nonalcoholic pediatric fatty liver disease: a multiethnic study. Hepatology. 2016;63:117-25.

80. Grandone A, Cozzolino D, Marzuillo P, Cirillo G, Di Sessa A, Ruggiero L, et al. TM6SF2 Glu167Lys polymorphism is associated with low levels of LDL-cholesterol and increased liver injury in obese children. Pediatr Obes. 2016;11:115-9.

81. Dongiovanni P, Petta S, Maglio C, Fracanzani AL, Pipitone R, Mozzi E, et al. Transmembrane 6 superfamily member 2 gene variant disentangles nonalcoholic steatohepatitis from cardiovascular disease. Hepatology. 2015;61:506-14.

82. Liu Z, Que S, Zhou L, Zheng S, Romeo S, Mardinoglu A, et al. The effect of the TM6SF2 E167K variant on liver steatosis and fibrosis in patients with chronic hepatitis C: a meta-analysis. Sci Rep. 2017;7:9273.

83. Liu DJ, Peloso GM, Yu H, Butterworth AS, Wang X, Mahajan A, et al. Exome-wide association study of plasma lipids in > 300, 000 individuals. Nat Genet. 2017;49:1758-66.

84. Holmen OL, Zhang H, Fan Y, Hovelson DH, Schmidt EM, Zhou W, et al. Systematic evaluation of coding variation identifies a candidate causal variant in TM6SF2 influencing total cholesterol and myocardial infarction risk. Nat Genet. 2014;46:345-51.

85. Chen LZ, Xia HHX, Xin YN, Lin ZH, Xuan SY. TM6SF2 E167K variant, a novel genetic susceptibility variant, contributing to nonalcoholic fatty liver disease. J Clin Transl Hepatol. 2015;3:265-70.

86. Viitasalo A, Pihlajamäki J, Paananen J, Atalay M, Lindi V, Lakka TA. Associations of TM6SF2 167K allele with liver enzymes and lipid profile in children: the PANIC study. Pediatr Res. 2016;79:684-8.

87. Pirola CJ, Sookoian S. The dual and opposite role of the TM6SF2-rs58542926 variant in protecting against cardiovascular disease and conferring risk for nonalcoholic fatty liver: a meta-analysis. Hepatology. 2015;62:1742-56.

88. Sookoian S, Castaño GO, Scian R, Mallardi P, Fernández Gianotti T, Burgueño AL, et al. Genetic variation in transmembrane 6 superfamily member 2 and the risk of nonalcoholic fatty liver disease and histological disease severity. Hepatology. 2015;61:515-25.

89. Musso G, Cipolla U, Cassader M, Pinach S, Saba F, De Michieli F, et al. TM6SF2 rs58542926 variant affects postprandial lipoprotein metabolism and glucose homeostasis in NAFLD. J Lipid Res. 2017;58:1221-9.

90. Chen F, Esmaili S, Rogers GB, Bugianesi E, Petta S, Marchesini G, et al. Lean NAFLD: a distinct entity shaped by differential metabolic adaptation. Hepatology. 2020;71:1213-27.

91. Liu YL, Reeves HL, Burt AD, Tiniakos D, McPherson S, Leathart JBS, et al. TM6SF2 rs58542926 influences hepatic fibrosis progression in patients with non-alcoholic fatty liver disease. Nat Commun. 2014;5:4309.

92. Eslam M, Mangia A, Berg T, Chan HL, Irving WL, Dore GJ, et al. Diverse impacts of the rs58542926 E167K variant in TM6SF2 on viral and metabolic liver disease phenotypes. Hepatology. 2016;64:34-46. 
93. Chen X, Zhou P, De L, Li B, Su S. The roles of transmembrane 6 superfamily member 2 rs58542926 polymorphism in chronic liver disease: a meta-analysis of 24, 147 subjects. Mol Genet Genomic Med. 2019;7:e824.

94. Stickel F, Buch S, Nischalke HD, Weiss KH, Gotthardt D, Fischer J, et al. Genetic variants in PNPLA3 and TM6SF2 predispose to the development of hepatocellular carcinoma in individuals with alcohol-related cirrhosis. Am J Gastroenterol. 2018;113:1475-83.

95. Petta S, Maida M, Grimaudo S, Pipitone RM, Macaluso FS, Cabibi D, et al. TM6SF2 rs58542926 is not associated with steatosis and fibrosis in large cohort of patients with genotype 1 chronic hepatitis $\mathrm{C}$. Liver Int. 2016;36:198-204.

96. Falleti E, Cussigh A, Cmet S, Fabris C, Toniutto P. PNPLA3 rs738409 and TM6SF2 rs58542926 variants increase the risk of hepatocellular carcinoma in alcoholic cirrhosis. Dig Liver Dis. 2016;48:69-75.

97. Yang J, Trépo E, Nahon P, Cao Q, Moreno C, Letouzé E, et al. PNPLA3 and TM6SF2 variants as risk factors of hepatocellular carcinoma across various etiologies and severity of underlying liver diseases. 2019;144:533-44.

98. Raksayot M, Chuaypen N, Khlaiphuengsin A, Pinjaroen N, Treeprasertsuk S, Poovorawan Y, et al. Independent and additive effects of PNPLA3 and TM6SF2 polymorphisms on the development of non-B, non-C hepatocellular carcinoma. J Gastroenterol. 2019;54:427-36.

99. Stickel F, Moreno C, Hampe J, Morgan MY. The genetics of alcohol dependence and alcohol-related liver disease. J Hepatol. 2017;66:195-211.

100. Buch S, Stickel F, Trépo E, Way M, Herrmann A, Nischalke HD, et al. A genome-wide association study confirms PNPLA3 and identifies TM6SF2 and MBOAT7 as risk loci for alcohol-related cirrhosis. Nat Genet. 2015;47:1443-8.

101. Mancina RM, Dongiovanni P, Petta S, Pingitore P, Meroni M, Rametta R, et al. The MBOAT7-TMC4 variant rs641738 increases risk of nonalcoholic fatty liver disease in individuals of European descent. Gastroenterology. 2016;150:1219-30.e6.

102. Luukkonen PK, Zhou Y, Hyötyläinen T, Leivonen M, Arola J, Orho-Melander M, et al. The MBOAT7 variant rs641738 alters hepatic phosphatidylinositols and increases severity of non-alcoholic fatty liver disease in humans. J Hepatol. 2016;65:1263-5.

103. Donati B, Dongiovanni P, Romeo S, Meroni M, McCain M, Miele L, et al. MBOAT7 rs641738 variant and hepatocellular carcinoma in non-cirrhotic individuals. Sci Rep. 2017;7:4492.

104. Viitasalo A, Eloranta AM, Atalay M, Romeo S, Pihlajamaki J, Lakka TA. Association of MBOAT7 gene variant with plasma ALT levels in children: the PANIC study. Pediatr Res. 2016;80:651-5.

105. Di Sessa A, Umano GR, Cirillo G, Del Prete A, Iacomino R, Marzuillo P, et al. The membrane-bound O-acyltransferase7 rs641738 variant in pediatric nonalcoholic fatty liver disease. J Pediatr Gastroenterol Nutr. 2018;67:69-74.

106. Krawczyk M, Rau M, Schattenberg JM, Bantel H, Pathil A, Demir M, et al. Combined effects of the PNPLA3 rs738409, TM6SF2 rs58542926, and MBOAT7 rs641738 variants on NAFLD severity: a multicenter biopsy-based study. J Lipid Res. 2017;58:247-55.

107. Thabet K, Chan HLY, Petta S, Mangia A, Berg T, Boonstra A, et al. The membrane-bound O-acyltransferase domain-containing 7 variant rs641738 increases inflammation and fibrosis in chronic hepatitis B. Hepatology. 2017;65:1840-50.

108. Thabet K, Asimakopoulos A, Shojaei M, Romero-Gomez M, Mangia A, Irving WL, et al. MBOAT7 rs641738 increases risk of liver inflammation and transition to fibrosis in chronic hepatitis C. Nat Commun. 2016;7:12757.

109. Rahal HK, Tabibian JH. The MBOAT7 rs641738 variant in primary sclerosing cholangitis: a novel biomarker for prognostication. Clin Res Hepatol Gastroenterol. 2020; [Epub ahead of print]. 
110. Freund C, Wahlers A, Begli NH, Leopold Y, Klöters-Plachky P, Mehrabi A, et al. The MBOAT7 rs641738 variant is associated with an improved outcome in primary sclerosing cholangitis. Clin Res Hepatol Gastroenterol. 2020; [Epub ahead of print].

111. Xia Y, Huang CX, Li GY, Chen KH, Han L, Tang L, et al. Meta-analysis of the association between MBOAT7 rs641738, TM6SF2 rs58542926 and nonalcoholic fatty liver disease susceptibility. Clin Res Hepatol Gastroenterol. 2019;43:533-41.

112. Sookoian S, Flichman D, Garaycoechea ME, Gazzi C, Martino JS, Castaño GO, et al. Lack of evidence supporting a role of TMC4-rs641738 missense variant-MBOAT7- intergenic downstream variant-in the susceptibility to nonalcoholic fatty liver disease. Sci Rep. 2018;8:5097.

113. Basyte-Bacevice V, Skieceviciene J, Valantiene I, Sumskiene J, Petrenkiene V, Kondrackiene J, et al. TM6SF2 and MBOAT7 gene variants in liver fibrosis and cirrhosis. Int J Mol Sci. 2019;20:1277.

114. Koo BK, Joo SK, Kim D, Bae JM, Park JH, Kim JH, et al. Additive effects of PNPLA3 and TM6SF2 on the histological severity of non-alcoholic fatty liver disease. J Gastroenterol Hepatol. 2018;33:1277-85.

115. Anstee QM, Darlay R, Cockell S, Meroni M, Govaere O, Tiniakos D, et al. Genome-wide association study of non-alcoholic fatty liver and steatohepatitis in a histologically-characterised cohort. J Hepatol. 2020; [Epub ahead of print].

116. Teo K, Abeysekera KW, Adams L, Aigner E, Banales JM, Banerjee R, et al. rs641738C >T near MBOAT7 is positively associated with liver fat, ALT, and histological severity of NAFLD: a meta-analysis. medRxiv 19013623 [Preprint]. 2020 [cited 2020 Feb 9]. Available from: https://www.medrxiv.org/ content/10.1101/19013623v3

117. Zarini S, Hankin JA, Murphy RC, Gijón MA. Lysophospholipid acyltransferases and eicosanoid biosynthesis in zebrafish myeloid cells. Prostaglandins Other Lipid Mediat. 2014;113-115:52-61.

118. Meroni M, Dongiovanni P, Longo M, Carli F, Baselli G, Rametta R, et al. Mboat7 down-regulation by hyperinsulinemia induces fat accumulation in hepatocytes. EBioMedicine. 2020;52:102658.

119. Petta S, Miele L, Bugianesi E, Camma C, Rosso C, Boccia S, et al. Glucokinase regulatory protein gene polymorphism affects liver fibrosis in non-alcoholic fatty liver disease. PLoS One. 2014;9:e87523.

120. Santoro N, Zhang CK, Zhao H, Pakstis AJ, Kim G, Kursawe R, et al. Variant in the glucokinase regulatory protein (GCKR) gene is associated with fatty liver in obese children and adolescents. Hepatology. 2012;55:781-9.

121. Speliotes EK, Yerges-Armstrong LM, Wu J, Hernaez R, Kim LJ, Palmer CD, et al. Genome-wide association analysis identifies variants associated with nonalcoholic fatty liver disease that have distinct effects on metabolic traits. PLoS Genet. 2011;7:e1001324.

122. Raimondo A, Rees MG, Gloyn AL. Glucokinase regulatory protein: complexity at the crossroads of triglyceride and glucose metabolism. Curr Opin Lipidol. 2015;26:88-95.

123. Beer NL, Tribble ND, McCulloch LJ, Roos C, Johnson PR, Orho-Melander M, et al. The P446L variant in GCKR associated with fasting plasma glucose and triglyceride levels exerts its effect through increased glucokinase activity in liver. Hum Mol Genet. 2009;18:4081-8.

124. Valenti L, Alisi A, Nobili V. Unraveling the genetics of fatty liver in obese children: additive effect of P446L GCKR and I148M PNPLA3 polymorphisms. Hepatology. 2012;55:661-3.

125. Santoro N, Caprio S, Pierpont B, Van Name M, Savoye M, Parks EJ. Hepatic de novo lipogenesis in obese youth is modulated by a common variant in the GCKR gene. J Clin Endocrinol Metab. 2015;100:E1125-32.

126. Tan HL, Zain SM, Mohamed R, Rampal S, Chin KF, Basu RC, et al. Association of glucokinase regulatory gene polymorphisms with risk and severity of non-alcoholic fatty liver disease: an interaction study with adiponutrin gene. J Gastroenterol. 2014;49:1056-64.

127. Kawaguchi T, Shima T, Mizuno M, Mitsumoto Y, Umemura A, Kanbara Y, et al. Risk estimation model for nonalcoholic fatty liver disease in the Japanese using multiple genetic markers. PLoS One. 2018;13:e0185490. 
128. Kozian DH, Barthel A, Cousin E, Brunnhöfer R, Anderka O, März W, et al. Glucokinase-activating GCKR polymorphisms increase plasma levels of triglycerides and free fatty acids, but do not elevate cardiovascular risk in the ludwigshafen risk and cardiovascular health study. Horm Metab Res. 2010;42:502-6.

129. Abul-Husn NS, Cheng X, Li AH, Xin Y, Schurmann C, Stevis P, et al. A protein-truncating HSD17B13 variant and protection from chronic liver disease. N Engl J Med. 2018;378:1096-106.

130. Horiguchi Y, Araki M, Motojima K. 17beta-Hydroxysteroid dehydrogenase type 13 is a liver-specific lipid droplet-associated protein. Biochem Biophys Res Commun. 2008;370:235-8.

131. Su W, Wang Y, Jia X, Wu W, Li L, Tian X, et al. Comparative proteomic study reveals 17beta-HSD13 as a pathogenic protein in nonalcoholic fatty liver disease. Proc Natl Acad Sci U S A. 2014;111:11437-42.

132. Adam M, Heikelä H, Sobolewski C, Portius D, Mäki-Jouppila J, Mehmood A, et al. Hydroxysteroid (17ß) dehydrogenase 13 deficiency triggers hepatic steatosis and inflammation in mice. FASEB J. 2018;32:3434-47.

133. Kozlitina J, Stender S, Hobbs HH, Cohen JC. HSD17B13 and chronic liver disease in blacks and hispanics. N Engl J Med. 2018;379:1876-7.

134. Pirola CJ, Garaycoechea M, Flichman D, Arrese M, Martino JS, Gazzi C, et al. Splice variant rs72613567 prevents worst histologic outcomes in patients with nonalcoholic fatty liver disease. J Lipid Res. 2019;60:176-85.

135. Gellert-Kristensen H, Nordestgaard BG, Tybjaerg-Hansen A, Stender S. High risk of fatty liver disease amplifies the alanine transaminase-lowering effect of a HSD17B13 variant. Hepatology. 2020;71:56-66.

136. Yang J, Trépo E, Nahon P, Cao Q, Moreno C, Letouzé E, et al. A 17-beta-hydroxysteroid dehydrogenase 13 variant protects from hepatocellular carcinoma development in alcoholic liver disease. Hepatology. 2019;70:231-40.

137. Luukkonen PK, Tukiainen T, Juuti A, Sammalkorpi H, Haridas PAN, Niemelä O, et al. Hydroxysteroid 17- $\beta$ dehydrogenase 13 variant increases phospholipids and protects against fibrosis in nonalcoholic fatty liver disease. JCI Insight. 2020;5:e132158.

138. Stender, S., etal., Relationship between Genetic Variation at PPP1R3B and Liver Glycogen and Triglyceride Levels. Hepatology, 2017.

139. Dongiovanni P, Meroni M, Mancina RM, Baselli G, Rametta R, Pelusi S, et al. Protein phosphatase 1 regulatory subunit $3 \mathrm{~B}$ gene variation protects against hepatic fat accumulation and fibrosis in individuals at high risk of nonalcoholic fatty liver disease. Hepatol Commun. 2018;2:666-75.

140. Mehta MB, Shewale SV, Sequeira RN, Millar JS, Hand NJ, Rader DJ. Hepatic protein phosphatase 1 regulatory subunit 3B (Ppp1r3b) promotes hepatic glycogen synthesis and thereby regulates fasting energy homeostasis. J Biol Chem. 2017;292:10444-54.

141. Dongiovanni P, Rametta R, Meroni M, Valenti L. The role of insulin resistance in nonalcoholic steatohepatitis and liver disease development--a potential therapeutic target? Expert Rev Gastroenterol Hepatol. 2016;10:229-42.

142. Dongiovanni P, Meroni M, Baselli GA, Bassani GA, Rametta R, Pietrelli A, et al. Insulin resistance promotes lysyl oxidase like 2 induction and fibrosis accumulation in non-alcoholic fatty liver disease. Clin Sci (Lond). 2017;131:1301-15.

143. Angulo P, Kleiner DE, Dam-Larsen S, Adams LA, Bjornsson ES, Charatcharoenwitthaya P, et al. Liver fibrosis, but no other histologic features, is associated with long-term outcomes of patients with nonalcoholic fatty liver disease. Gastroenterology. 2015;149:389-97.e10.

144. McPherson S, Hardy T, Henderson E, Burt AD, Day CP, Anstee QM. Evidence of NAFLD progression from steatosis to fibrosing-steatohepatitis using paired biopsies: implications for prognosis and clinical management. J Hepatol. 2015;62:1148-55. 
145. Pelusi S, Petta S, Rosso C, Borroni V, Fracanzani AL, Dongiovanni P, et al. Renin-angiotensin system inhibitors, type 2 diabetes and fibrosis progression: an observational study in patients with nonalcoholic fatty liver disease. PLoS One. 2016;11:e0163069.

146. Dongiovanni P, Valenti L, Rametta R, Daly AK, Nobili V, Mozzi E, et al. Genetic variants regulating insulin receptor signalling are associated with the severity of liver damage in patients with non-alcoholic fatty liver disease. Gut. 2010;59:267-73.

147. Kitamoto A, Kitamoto T, Nakamura T, Ogawa Y, Yoneda M, Hyogo H, et al. Association of polymorphisms in GCKR and TRIB1 with nonalcoholic fatty liver disease and metabolic syndrome traits. Endocr J. 2014;61:683-9.

148. Cefalù AB, Pirruccello JP, Noto D, Gabriel S, Valenti V, Gupta N, et al. A novel APOB mutation identified by exome sequencing cosegregates with steatosis, liver cancer, and hypocholesterolemia. Arterioscler Thromb Vasc Biol. 2013;33:2021-5.

149. Pelusi S, Baselli G, Pietrelli A, Dongiovanni P, Donati B, McCain MV, et al. Rare pathogenic variants predispose to hepatocellular carcinoma in nonalcoholic fatty liver disease. Sci Rep. 2019;9:3682.

150. Di Filippo M, Moulin P, Roy P, Samson-Bouma ME, Collardeau-Frachon S, Chebel-Dumont S, et al. Homozygous MTTP and APOB mutations may lead to hepatic steatosis and fibrosis despite metabolic differences in congenital hypocholesterolemia. J Hepatol. 2014;61:891-902.

151. Petersen KF, Dufour S, Hariri A, Nelson-Williams C, Foo JN, Zhang XM, et al. Apolipoprotein C3 gene variants in nonalcoholic fatty liver disease. N Engl J Med. 2010;362:1082-9.

152. Kozlitina J, Boerwinkle E, Cohen JC, Hobbs HH. Dissociation between APOC3 variants, hepatic triglyceride content and insulin resistance. Hepatology. 2011;53:467-74.

153. Valenti L, Nobili V, Al-Serri A, Rametta R, Leathart JB, Zappa MA, et al. The APOC3 T-455C and C-482T promoter region polymorphisms are not associated with the severity of liver damage independently of PNPLA3 I148M genotype in patients with nonalcoholic fatty liver. J Hepatol. 2011;55:1409-14.

154. Dongiovanni P, Meroni M, Baselli G, Mancina RM, Ruscica M, Longo M, et al. PCSK7 gene variation bridges atherogenic dyslipidemia with hepatic inflammation in NAFLD patients. J Lipid Res. 2019;60:1144-53.

155. Huang T, Huang J, Qi Q, Li Y, Bray GA, Rood J, et al. PCSK7 genotype modifies effect of a weight-loss diet on 2-year changes of insulin resistance: the POUNDS LOST trial. Diabetes Care. 2015;38:439-44.

156. Cohen J, Pertsemlidis A, Kotowski IK, Graham R, Garcia CK, Hobbs HH. Low LDL cholesterol in individuals of African descent resulting from frequent nonsense mutations in PCSK9. Nat Genet. 2005;37:161-5.

157. Ruscica M, Ferri N, Macchi C, Meroni M, Lanti C, Ricci C, et al. Liver fat accumulation is associated with circulating PCSK9. Ann Med. 2016;48:384-91.

158. Trinder M, Francis GA, Brunham LR. Association of monogenic vs. polygenic hypercholesterolemia with risk of atherosclerotic cardiovascular disease. JAMA Cardiol. 2020; [Epub ahead of print].

159. Costet P, Cariou B, Lambert G, Lalanne F, Lardeux B, Jarnoux AL, et al. Hepatic PCSK9 expression is regulated by nutritional status via insulin and sterol regulatory element-binding protein $1 \mathrm{c}$. J Biol Chem. 2006;281:6211-8.

160. Kotowski IK, Pertsemlidis A, Luke A, Cooper RS, Vega GL, Cohen JC, et al. A spectrum of PCSK9 alleles contributes to plasma levels of low-density lipoprotein cholesterol. Am J Hum Genet. 2006;78:410-22.

161. Gomaraschi M, Fracanzani AL, Dongiovanni P, Pavanello C, Giorgio E, Da Dalt L, et al. Lipid accumulation impairs lysosomal acid lipase activity in hepatocytes: evidence in NAFLD patients and cell cultures. Biochim Biophys Acta Mol Cell Biol Lipids. 2019;1864:158523.

162. Reiner Ž, Guardamagna O, Nair D, Soran H, Hovingh K, Bertolini S, etal. Lysosomal acid lipase deficiency--an under-recognized cause of dyslipidaemia and liver dysfunction. Atherosclerosis. 2014;235:21-30.

163. Auinger A, Valenti L, Pfeuffer M, Helwig U, Herrmann J, Fracanzani AL, et al. A promoter polymorphism in the liver-specific fatty acid transport protein 5 is associated with features of the metabolic syndrome and steatosis. Horm Metab Res. 2010;42:854-9. 
164. Valenti L, Motta BM, Alisi A, Sartorelli R, Buonaiuto G, Dongiovanni P, et al. LPIN1 rs13412852 polymorphism in pediatric nonalcoholic fatty liver disease. J Pediatr Gastroenterol Nutr. 2012;54:588-93.

165. Marra F, Bertolani C. Adipokines in liver diseases. Hepatology. 2009;50:957-69.

166. Miele L, Valenza V, La Torre G, Montalto M, Cammarota G, Ricci R, et al. Increased intestinal permeability and tight junction alterations in nonalcoholic fatty liver disease. Hepatology. 2009;49:1877-87.

167. Bilzer M, Roggel F, Gerbes AL. Role of Kupffer cells in host defense and liver disease. Liver Int. 2006;26:1175-86.

168. Eslam M, McLeod D, Kelaeng KS, Mangia A, Berg T, Thabet K, et al. IFN- $\lambda 3$, not IFN- $\lambda 4$, likely mediates IFNL3-IFNL4 haplotype-dependent hepatic inflammation and fibrosis. Nat Genet. 2017;49:795-800.

169. PettaS, Grimaudo S, Cammà C, Cabibi D, Di Marco V, Licata G, et al. IL28B and PNPLA3 polymorphisms affect histological liver damage in patients with non-alcoholic fatty liver disease. J Hepatol. 2012;56:1356-62.

170. Eslam M, Hashem AM, Leung R, Romero-Gomez M, Berg T, Dore GJ, et al. Interferon- $\lambda$ rs12979860 genotype and liver fibrosis in viral and non-viral chronic liver disease. Nat Commun. 2015;6:6422.

171. Eslam M, Hashem AM, Romero-Gomez M, Berg T, Dore GJ, Mangia A, et al. FibroGENE: a gene-based model for staging liver fibrosis. J Hepatol. 2016;64:390-8.

172. Petta S, Valenti L, Tuttolomondo A, Dongiovanni P, Pipitone RM, Cammà C, et al. Interferon lambda 4 rs368234815 TT $>\delta G$ variant is associated with liver damage in patients with nonalcoholic fatty liver disease. Hepatology. 2017;66:1885-93.

173. Petta S, Valenti L, Svegliati-Baroni G, Ruscica M, Pipitone RM, Dongiovanni P, et al. Fibronectin type III domain-containing protein 5 rs3480 A>G polymorphism, irisin, and liver fibrosis in patients with nonalcoholic fatty liver disease. J Clin Endocrinol Metab. 2017;102:2660-9.

174. Metwally M, Bayoumi A, Romero-Gomez M, Thabet K, John M, Adams LA, et al. A polymorphism in the Irisin-encoding gene (FNDC5) associates with hepatic steatosis by differential miRNA binding to the 3’UTR. J Hepatol. 2019;70:494-500.

175. Gorden A, Yang R, Yerges-Armstrong LM, Ryan KA, Speliotes E, Borecki IB, et al. Genetic variation at NCAN locus is associated with inflammation and fibrosis in non-alcoholic fatty liver disease in morbid obesity. Hum Hered. 2013;75:34-43.

176. Cai W, Weng DH, Yan P, Lin YT, Dong ZH, Mailamuguli, et al. Genetic polymorphisms associated with nonalcoholic fatty liver disease in Uyghur population: a case-control study and meta-analysis. Lipids Health Dis. 2019;18:14.

177. Cicione C, Degirolamo C, Moschetta A. Emerging role of fibroblast growth factors $15 / 19$ and 21 as metabolic integrators in the liver. Hepatology. 2012;56:2404-11.

178. Dongiovanni P, Crudele A, Panera N, Romito I, Meroni M, De Stefanis C, et al. beta-Klotho gene variation is associated with liver damage in children with NAFLD. J Hepatol. 2020;72:411-9.

179. Petta S, Valenti L, Marra F, Grimaudo S, Tripodo C, Bugianesi E, et al. MERTK rs4374383 polymorphism affects the severity of fibrosis in non-alcoholic fatty liver disease. J Hepatol. 2016;64:682-90.

180. Rüeger S, Bochud PY, Dufour JF, Müllhaupt B, Semela D, Heim MH, et al. Impact of common risk factors of fibrosis progression in chronic hepatitis C. Gut. 2015;64:1605-15.

181. Cai B, Dongiovanni P, Corey KE, Wang X, Shmarakov IO, Zheng Z, et al. Macrophage MerTK Promotes Liver Fibrosis in Nonalcoholic Steatohepatitis. Cell Metab. 2020;31:406-21.e7.

182. Miele L, Beale G, Patman G, Nobili V, Leathart J, Grieco A, et al. The Kruppel-like factor 6 genotype is associated with fibrosis in nonalcoholic fatty liver disease. Gastroenterology. 2008;135:282-91.e1.

183. Rametta R, Meroni M, Dongiovanni P. From environment to genome and back: a lesson from HFE mutations. Int J Mol Sci. 2020;21:3505. 
184. Aravinthan A, Mells G, Allison M, Leathart J, Kotronen A, Yki-Jarvinen H, et al. Gene polymorphisms of cellular senescence marker p21 and disease progression in non-alcohol-related fatty liver disease. Cell Cycle. 2014;13:1489-94.

185. Calado RT, Regal JA, Kleiner DE, Schrump DS, Peterson NR, Pons V, et al. A spectrum of severe familial liver disorders associate with telomerase mutations. PLoS One. 2009;4:e7926.

186. Pessayre D, Fromenty B. NASH: a mitochondrial disease. J Hepatol. 2005;42:928-40.

187. Mansouri A, Gattolliat CH, Asselah T. Mitochondrial dysfunction and signaling in chronic liver diseases. Gastroenterology. 2018;155:629-47.

188. Al-Serri A, Anstee QM, Valenti L, Nobili V, Leathart JB, Dongiovanni P, et al. The SOD2 C47T polymorphism influences NAFLD fibrosis severity: evidence from case-control and intra-familial allele association studies. J Hepatol. 2012;56:448-54.

189. Namikawa C, Shu-Ping Z, Vyselaar JR, Nozaki Y, Nemoto Y, Ono M, et al. Polymorphisms of microsomal triglyceride transfer protein gene and manganese superoxide dismutase gene in non-alcoholic steatohepatitis. J Hepatol. 2004;40:781-6.

190. Serviddio G, Bellanti F, Tamborra R, Rollo T, Capitanio N, Romano AD, et al. Uncoupling protein-2 (UCP2) induces mitochondrial proton leak and increases susceptibility of non-alcoholic steatohepatitis (NASH) liver to ischaemia-reperfusion injury. Gut. 2008;57:957-65.

191. Fares R, Petta S, Lombardi R, Grimaudo S, Dongiovanni P, Pipitone R, et al. The UCP2 -866 G>A promoter region polymorphism is associated with nonalcoholic steatohepatitis. Liver Int. 2015;35:1574-80.

192. Aller R, De Luis DA, Izaola O, González Sagrado M, Conde R, Alvarez T, et al. Role of -55CT polymorphism of UCP3 gene on non alcoholic fatty liver disease and insulin resistance in patients with obesity. Nutr Hosp. 2010;25:572-6.

193. Dong C, Della-Morte D, Wang L, Cabral D, Beecham A, McClendon MS, et al. Association of the sirtuin and mitochondrial uncoupling protein genes with carotid plaque. PLoS One. 2011;6:e27157.

194. Emdin CA, Haas ME, Khera AV, Aragam K, Chaffin M, Klarin D, et al. A missense variant in mitochondrial amidoxime reducing component 1 gene and protection against liver disease. PLoS Genet. 2020;16:e1008629.

195. Trépo E,Valenti L. Update on NAFLD genetics: from new variants to the clinic. J Hepatol. 2020;72:1196-209.

196. Di Costanzo A, Pacifico L, Chiesa C, Perla FM, Ceci F, Angeloni A, et al. Genetic and metabolic predictors of hepatic fat content in a cohort of Italian children with obesity. Pediatr Res. 2019;85:671-7.

197. Suomela E, Oikonen M, Pitkänen N, Ahola-Olli A, Virtanen J, Parkkola R, et al. Childhood predictors of adult fatty liver. The cardiovascular risk in young finns study. J Hepatol. 2016;65:784-90.

198. Chen L, Du S, Lu L, Lin Z, Jin W, Hu D, et al. The additive effects of the TM6SF2 E167K and PNPLA3 I148M polymorphisms on lipid metabolism. Oncotarget. 2017;8:74209-16.

199. Gellert-Kristensen H, Richardson TG, Davey Smith G, Nordestgaard BG, Tybjaerg-Hansen A, Stender S. Combined effect of PNPLA3, TM6SF2, and HSD17B13 variants on risk of cirrhosis and hepatocellular carcinoma in the general population. Hepatology. 2020; [Epub ahead of print].

200. Eslam M, George J. Genetic Insights for Drug Development in NAFLD. Trends Pharmacol Sci. 2019;40:506-16. 